\title{
Operator-valued kernel-based vector autoregressive models for network inference
}

\author{
Néhémy Lim • Florence d'Alché-Buc • Cédric Auliac • \\ George Michailidis
}

Received: 3 March 2013 / Accepted: 25 November 2014 / Published online: 17 December 2014

(C) The Author(s) 2014

\begin{abstract}
Reverse-engineering of high-dimensional dynamical systems from time-course data still remains a challenging and important problem in knowledge discovery. For this learning task, a number of approaches primarily based on sparse linear models or Granger causality concepts have been proposed in the literature. However, when a system exhibits nonlinear dynamics, there does not exist a systematic approach that takes into account the nature of the underlying system. In this work, we introduce a novel family of vector autoregressive models based on different operator-valued kernels to identify the dynamical system and retrieve the target network that characterizes the interactions of its components. Assuming a sparse underlying structure, a key challenge, also present in the linear case, is to control the model's sparsity. This is achieved through the joint learning of the structure of the kernel and the basis vectors. To solve this learning task, we propose an alternating optimization algorithm based on proximal gradient procedures that learns both the structure of the ker-
\end{abstract}

Electronic supplementary material The online version of this article (doi:10.1007/s10994-014-5479-3) contains supplementary material, which is available to authorized users.

Editors: Toon Calders, Rosa Meo, Floriana Esposito, and Eyke Hullermeier.

N. Lim · C. Auliac

CEA, LIST, 91191 Gif-sur-Yvette Cedex, France

e-mail: nehemy.lim@cea.fr

C. Auliac

e-mail: cedric.auliac@cea.fr

N. Lim · F. d'Alché-Buc $(\varangle)$

IBISC EA 4526, Université d'Évry-Val d'Essonne, 91000 Évry, France

e-mail: florence.dalche@ibisc.univ-evry.fr; florence.dalche@telecom-paristech.fr

F. d'Alché-Buc

INRIA-Saclay, TAO, LRI umr CNRS 8623, Université Paris Sud, Paris, France

G. Michailidis

Department of Statistics, University of Michigan, Ann Arbor, MI 48109-1107, USA

e-mail: gmichail@umich.edu 
nel and the basis vectors. Results on the DREAM3 competition gene regulatory benchmark networks of sizes 10 and 100 show the new model outperforms existing methods. Another application of the model on climate data identifies interesting and interpretable interactions between natural and human activity factors, thus confirming the ability of the learning scheme to retrieve dependencies between state-variables.

Keywords Network inference - Operator-valued kernel · Regularization · Proximal gradient methods $\cdot$ Vector autoregressive model $\cdot$ Jacobian

\section{Introduction}

In many scientific problems, high dimensional data with network structure play a key role in knowledge discovery (Kolaczyk 2009). For example, recent advances in high throughput technologies have facilitated the simultaneous study of components of complex biological systems. Hence, molecular biologists are able to measure the expression levels of the entire genome and a good portion of the proteome and metabolome under different conditions and thus gain insight on how organisms respond to their environment. For this reason, reconstruction of gene regulatory networks from expression data has become a canonical problem in computational systems biology (Lawrence et al. 2010). Similar data structures emerge in other scientific domains. For instance, political scientists have focused on the analysis of roll call data of legislative bodies, since they allow them to study party cohesion and coalition formation through the underlying network reconstruction (Morton and Williams 2010), while economists have focused on understanding companies' creditworthiness or contagion (Gilchrist et al. 2009). Understanding climate changes implies to be able to predict the behavior of climate variables and their dependence relationship (Parry et al. 2007; Liu et al. 2010). Two classes of network inference problems have emerged simultaneously from all these fields: the inference of association networks that represent coupling between variables of interest (Meinshausen and Bühlmann 2006; Kramer et al. 2009) and the inference of "causal" networks that describe how variables influence each other (Murphy 1998; Perrin et al. 2003; Auliac et al. 2008; Zou and Feng 2009; Shojaie and Michailidis 2010; Maathuis et al. 2010; Bolstad et al. 2011; Dondelinger et al. 2013; Chatterjee et al. 2012).

Over the last decade, a number of statistical techniques have been introduced for estimating networks from high-dimensional data in both cases. They divide into model-free and model-driven approaches. Model-free approaches for association networks directly estimate information-theoretic measures, such as mutual information to detect edges in the network (Hartemink 2005; Margolin et al. 2006). Among model-driven approaches, graphical models have emerged as a powerful class of models and a lot of algorithmic and theoretical advances have occured for static (independent and identically distributed) data under the assumption of sparsity. For instance, Gaussian graphical models have been thoroughly studied (see Bühlmann and van de Geer (2011) and references therein) under different regularization schemes to reinforce sparsity in linear models in an unstructured or a structured way. In order to infer causal relationship networks, Bayesian networks (Friedman 2004; Lèbre 2009) have been developed either from static or time-series data within the framework of dynamical Bayesian networks. In the case of continuous variables, linear multivariate autoregressive modeling (Michailidis and d'Alché Buc 2013) has been developed with again an important focus on sparse models. In this latter framework, Granger causality models have attracted an increasing interest to capture causal relationships. 
However, to date, few papers in the literature have focused on network inference for continuous variables in the presence of nonlinear dynamics despite the fact that many mechanisms (e.g. regulatory ones in biology) involve such dynamics. Of special interest are approaches based on parametric ordinary differential equations (Chou and Voit 2009) that alternatively learn the structure of the model and its parameters. The most successful approaches are based on Bayesian Learning (Mazur et al. 2009; Aijo and Lahdesmaki 2009) that allow them to deal with the stochasticity of biological data, while easily incorporating prior knowledge and genetic programming (Iba 2008) that provide a population-based algorithm for a stochastic search in the structure space. In this study, we start from a regularization theory perspective and introduce a general framework for nonlinear multivariate modeling and network inference. Our aim is to extend the framework of sparse linear modeling to that of sparse nonlinear modeling. In the machine learning community, a powerful tool to extend linear models to nonlinear ones is based on kernels. The famous "kernel trick" allows one to deal with nonlinear learning problems by working implicitly in a new feature space, where inner products can be computed using a symmetric positive semi-definite function of two variables, called a kernel. In particular, a given kernel allows to build a unique Reproducing Kernel Hilbert Space (RKHS), e.g. a functional space where regularized models can be defined from data using representer theorems. The RKHS theory provides a unified framework for many kernel-based models and a principled way to build new (nonlinear) models. Since multivariate time-series modeling requires defining vector-valued models, we propose to build on operator-valued kernels and their associated reproducing kernel Hilbert space theory (Senkene and Tempel'man 1973) that were introduced in machine learning by Micchelli and Pontil (2005) for the multi-task learning problem with vector-valued functions. This is an active area-see review (Alvarez et al. 2011)—with new applications on vector field regression (Baldassarre et al. 2010), structured classification (Dinuzzo and Fukumizu 2011), functional regression (Kadri et al. 2011) and link prediction (Brouard et al. 2011). However, the use of operator-valued kernels in the context of time series is novel.

Building upon our previous work (Lim et al. 2013) that focused on a specific model, we define a whole family of nonlinear vector autoregressive models based on various operatorvalued kernels. Once an operator-valued kernel-based model is learnt, we compute an empirical estimate of its Jacobian, providing a generic and simple way to extract dependence relationships among variables. We discuss how a specific operator-valued kernel can produce not only a good approximation of the system dynamics, but also a flexible and controllable Jacobian estimate. To obtain sparse networks and get sparse Jacobian estimates, we extend the sparsity constraint regularly used in linear modeling.

To control smoothness of the model, the definition of the loss function involves an $\ell_{2}$-norm penalty and additionally, may include two different types of penalties, either an $\ell_{1}$-norm penalty or a mixed $\ell_{1} / \ell_{2}$-norm regularization applied to the matrix of parameter vectors, depending on the nature of the estimation problem. To optimize a loss function that contains these non-differentiable terms, we develop a general proximal gradient algorithm.

Note that selected operator-valued kernels involve a positive semi-definite matrix as a hyperparameter. The background knowledge required for its definition is in general not available, especially in a network inference task. To address this kernel design task together with learning the other parameters, we introduce an efficient strategy that alternatively learns the parameter vectors and the positive semi-definite matrix that characterizes the kernel. This matrix plays an important role regarding the Jacobian sparsity; the estimation procedure for the matrix parameter also involves an $\ell_{1}$ penalty and a positive semi-definiteness constraint. We show that without prior knowledge on the relationship between variables, the proposed 
algorithm is able to retrieve the network structure of a given underlying dynamical system from the observation of its behavior through time.

The remainder of the paper is organized as follows: in Sect. 2, we present the general network inference scheme. In Sect. 3, we recall elements of RKHS theory devoted to vectorvalued functions and introduce operator-valued kernel-based autoregressive models. Section 4 presents the learning algorithm that estimates both the parameters of the model and the parameters of the kernel. Section 5 illustrates the performance of the model and the algorithm through extensive numerical work based on both synthetic and real data, and comparison with state-of-the-art methods.

\section{Network inference from nonlinear vector autoregressive models}

Let $\mathbf{x}_{t} \in \mathbb{R}^{d}$ denote the observed state of a dynamical system comprising $d$ state variables. We are interested in inferring direct influences of a state variable $j$ on other variables $i \neq$ $j,(i, j) \in\{1, \ldots, d\}^{2}$. The set of influences among state variables is encoded by a network matrix $A=\left(a_{i j}\right)$ of size $d \times d$ for which a coefficient $a_{i j}=1$ if the state variable $j$ influences the state variable $i$, and 0 otherwise. Further, we assume that a first-order stationary model is adequate to capture the temporal evolution of the system under study, which can exhibit nonlinear dynamics captured by a function $h: \mathbb{R}^{d} \rightarrow \mathbb{R}^{d}$ :

$$
\mathbf{x}_{t+1}=h\left(\mathbf{x}_{t}\right)+\mathbf{u}_{t+1}
$$

where $\mathbf{u}_{t+1}$ is a noise term. Let us call $\mathbf{x}_{1}, \ldots, \mathbf{x}_{N+1}$ the observed time series of the network states at regular spaced time points. $\mathbf{x}_{1}$ denotes the initial condition and the whole evolution of the model depends on it.

Linear models $\left(h\left(\mathbf{x}_{t}\right)=B \mathbf{x}_{t}\right)$ or other parametric models, explicitly involve a matrix that can be interpreted as a network matrix and its estimation (possibly sparse) can be directly accomplished. However, for nonlinear models this is a more involved task. Our strategy is to first learn $h$ from the data and subsequently estimate $A$ using the values of the instantaneous Jacobian matrix of model $h$, measured at each time point. The underlying idea is that partial derivatives $\frac{\partial h\left(\mathbf{x}_{t}\right)^{i}}{\partial x_{t}^{j}}$ reflect the influence of explanatory variable $j$ at time $t$ on the value of the $i$ th model's output $h\left(\mathbf{x}_{t}\right)^{i}$. If $\frac{\partial h\left(\mathbf{x}_{t}\right)^{i}}{\partial x_{t}^{j}}$ is high in absolute value, then variable $j$ influences variable $i$. Several estimators of $A$ can be built from these partial derivatives. We propose to use the empirical mean of the instantaneous Jacobian matrix of $h$ using the time series $\mathbf{x}_{1}, \ldots, \mathbf{x}_{N+1}$. Then, $\forall(i, j) \in\{1, \ldots, d\}^{2}$, an estimate $\hat{J}(h)$ of the Jacobian matrix $\nabla h=J(h)$ is given by:

$$
\hat{J}(h)_{i j}=\frac{1}{N} \sum_{t=1}^{N} \frac{\partial h\left(\mathbf{x}_{t}\right)^{i}}{\partial x_{t}^{j}}
$$

In the remainder of the paper, we note $\hat{J}(h)_{i j}$ the $(i, j)$ coefficient of the empirical mean of Jacobian of $h$ and $\hat{J}(h)_{i j}(t)$ its value at a given time $t$. Each coefficient $\hat{J}(h)_{i j}$ in absolute value gives a score to the potential influence of variable $j$ on variable $i$. To provide a final estimate of $A$, these coefficients are sorted and thresholded.

Note that to obtain a high quality estimate of the network, we need a class of functions $h$ whose Jacobian matrices can be controlled during learning in such a way that they could provide good continuous approximators of $A$. In this work, we propose a new class of nonparametric vector autoregressive models that exhibit such properties. Specifically, we introduce Operator-valued Kernel-based Vector AutoRegressive (OKVAR) models, that constitute a rich class as discussed in the next section. 


\section{Operator-valued kernels and vector autoregressive models}

\subsection{From scalar-valued kernel to operator-valued kernel models of autoregresssion}

In order to solve the vector autoregression problem set in Eq. (1) with a nonlinear model, one option is to decompose it into $d$ tasks and use, for each task $i$, a scalar-valued model $h_{i}$ such as a kernel-based model. Dataset $\mathscr{D}_{N}=\left\{\left(\mathbf{x}_{\ell}, \mathbf{x}_{\ell+1}\right), \ell=1, \ldots, N\right\}$ now reduces into $d$ datasets of the form $\mathscr{D}_{N}^{i}=\left\{\left(\mathbf{x}_{\ell}, x_{\ell+1}^{i}\right), \ell=1, \ldots, N\right\}$. Each task $i$ is now a regression one that we can solve by estimating a nonparametric model. For instance, kernel-based regression models are good candidates for such tasks. A unique feature of these approaches is that they can be derived from Reproducing Kernel Hilbert Space theory that offers a rigorous background for regularization. For instance, kernel-ridge regression and Support Vector Regression provide consistent estimators for the model, as long as the chosen kernel is universal. Then, for each $i=1, \ldots, d$, a model based on a positive definite kernel $k: \mathbb{R}^{d} \times \mathbb{R}^{d} \rightarrow \mathbb{R}$ can be written as:

$$
\hat{x}_{t+1}^{i}=h_{i}\left(\mathbf{x}_{t}\right)=\sum_{\ell=1}^{N} w_{\ell}^{i} k\left(\mathbf{x}_{\ell}, \mathbf{x}_{t}\right),
$$

where $\mathbf{w}^{i}$ is the parameter vector attached to model $i$. Although this decomposition is well justified when the covariance matrix of noise $\mathbf{u}_{t}$ is diagonal, in the general case, the $d$ regression tasks are not independent or even uncorrelated. The purpose of this work is therefore to extend such approaches to the vector autoregression problem in order to provide (i) a general family of nonparametric models, and (ii) suitable models for network inference by Jacobian estimation. We now aim to predict the state vector of a dynamical system $\mathbf{x}_{t+1}$ at time $t+1$, given its state $\mathbf{x}_{t}$ at time $t$ using kernel-based models appropriate for vectors. As a vector autoregressive model is a vector-valued function, the RKHS theory based on scalar-valued kernels does not apply. However, if the kernel is chosen to be operator-valued e.g. matrix-valued in our setting, then RKHS theory devoted to operator-valued kernels provides a similar framework to build models and to justify their use. In the following, we introduce the basic building blocks of operatorvalued kernel-based theory and notations to extend (3) into models of the following form:

$$
\hat{\mathbf{x}}_{t+1}=h\left(\mathbf{x}_{t}\right)=\sum_{\ell=1}^{N} K\left(\mathbf{x}_{t}, \mathbf{x}_{\ell}\right) \mathbf{w}_{\ell},
$$

where $K$ is an operator-valued kernel to be defined in next section and $\mathbf{w}_{\ell}, \ell=1, \ldots, N$ are parameter vectors of dimension $d$.

\subsection{Basics of operator-valued kernel-based theory}

In RKHS theory with operator-valued kernels, we consider functions with input in some set $\mathscr{X}$ and output with vector values in some given Hilbert space $\mathscr{F} y$. For completeness, we first describe the general framework and then come back to the case of interest, namely $\mathscr{X}=\mathscr{F}_{y}=\mathbb{R}^{d}$. Denote by $L\left(\mathscr{F}_{y}\right)$, the set of all bounded linear operators from $\mathscr{F}_{y}$ to itself. Given $A \in L\left(\mathscr{F}_{y}\right), A^{*}$ denotes its adjoint. Then, an operator-valued kernel $K$ is defined as follows:

Definition 1 (Operator-valued kernel Senkene and Tempel'man 1973; Caponnetto et al. 2008) Let $\mathscr{X}$ be a set and $\mathscr{F}_{y}$ a Hilbert space. Then, $K: \mathscr{X} \times \mathscr{X} \rightarrow L\left(\mathscr{F}_{y}\right)$ is a kernel if:

$-\forall(\mathbf{x}, \mathbf{z}) \in \mathscr{X} \times \mathscr{X}, K(\mathbf{x}, \mathbf{z})=K(\mathbf{z}, \mathbf{x})^{*}$

- $\forall m \in \mathbb{N}, \forall\left\{\left(\mathbf{x}_{i}, \mathbf{y}_{i}\right), i=1, \ldots, m\right\} \subseteq \mathscr{X} \times \mathscr{F} y, \sum_{i, j=1}^{m}\left\langle\mathbf{y}_{i}, K\left(\mathbf{x}_{i}, \mathbf{x}_{j}\right) \mathbf{y}_{j}\right\rangle_{\mathscr{F}_{y}} \geq 0$ 
Let us consider the case of regression, we denote by $\mathscr{D}_{N}=\left\{\left(\mathbf{x}_{\ell}, \mathbf{y}_{\ell}\right), \ell=1, \ldots, N\right\} \subseteq$ $\mathscr{X} \times \mathscr{F}_{y}$ the data set under consideration. We are interested in a new family of models of the form:

$$
h(\cdot)=\sum_{\ell=1}^{N} K\left(\cdot, \mathbf{x}_{\ell}\right) \mathbf{c}_{\ell}
$$

where the coefficients $\mathbf{c}_{\ell}, \ell=\{1, \ldots, N\}$ are vectors in the Hilbert space $\mathscr{F} y$. For vector regression in $\mathbb{R}^{d}$, the operator-valued kernel (OVK) becomes a matrix-valued one. If this matrix is diagonal, the model reduces to $d$ independent models with scalar outputs and there is no need for a matrix-valued kernel. In other cases, when we assume that the different components of the vector-valued function are not independent and may share some underlying structure, a non-diagonal matrix-valued kernel allows to take into consideration dependencies between the components of the input vectors. Initial applications of matrix-valued kernels deal with structured output regression tasks, such as multi-task learning (Micchelli and Pontil 2005), structured classification (Dinuzzo and Fukumizu 2011) and link prediction (Brouard et al. 2011). In the following, we propose to apply this framework to autoregression. We examine different matrix-valued kernels as well as different loss functions and discuss their relevance for network inference.

\subsection{The OKVAR family}

Let us now fix $\mathscr{X}=\mathscr{F}_{y}=\mathbb{R}^{d}$. Recall that the objective is to estimate a vector autoregressive model. Given the observed $d$-dimensional time series $\mathbf{x}_{1}, \ldots, \mathbf{x}_{N+1}$ that we use as the training dataset $\mathscr{D}_{N}=\left\{\left(\mathbf{x}_{1}, \mathbf{x}_{2}\right), \ldots,\left(\mathbf{x}_{N}, \mathbf{x}_{N+1}\right)\right\}$, the nonparametric model $h$ is defined as

$$
\hat{\mathbf{x}}_{t+1}=h\left(\mathbf{x}_{t} ; \mathscr{D}_{N}\right)=\sum_{\ell=1}^{N} K\left(\mathbf{x}_{t}, \mathbf{x}_{\ell}\right) \mathbf{c}_{\ell}
$$

where $K(\cdot, \cdot)$ is a matrix-valued kernel and each $\mathbf{c}_{\ell}(\ell \in\{1, \ldots, N\})$ is a vector of dimension $d$. In the following, we denote by $C \in \mathscr{M}^{d, N}$, the matrix composed of the $N$ column vectors $\mathbf{c}_{\ell}$ of dimension $d$. We call Operator-valued Kernel Vector Autoregression (OKVAR), the vector autoregressive models of the form given by Eq. (6). In this study, we focus on nonlinear kernels by considering three kernel candidates that fulfill the properties of an operator-valued kernel, one of them being universal.

Let us recall the definition of the scalar-valued Gaussian kernel $k_{\text {Gauss }}: \mathbb{R}^{d} \times \mathbb{R}^{d} \rightarrow \mathbb{R}$ : $k_{\text {Gauss }}(\mathbf{x}, \mathbf{z})=\exp \left(-\gamma\|\mathbf{x}-\mathbf{z}\|^{2}\right)$. Please notice that in the special case $d=1, k_{\text {Gauss }}(x, z)$ reduces to $\exp \left(-\gamma(x-z)^{2}\right)$.

As a baseline, we first consider the Gaussian transformable kernel which extends the standard Gaussian kernel to the matrix-valued case. If $\mathbf{x}$ is a vector, we denote $x^{m}$ its $m$ th coordinate. Then the Gaussian transformable kernel is defined as follows:

Definition 2 (Gaussian (transformable) kernel)

$$
\forall(\mathbf{x}, \mathbf{z}) \in \mathbb{R}^{d} \times \mathbb{R}^{d}, \forall(i, j) \in\{1, \ldots, d\}^{2}, K_{\text {Gauss }}(\mathbf{x}, \mathbf{z})_{i j}=k_{\text {Gauss }}\left(x^{i}, z^{j}\right)
$$

Interestingly, each $(i, j)$-coefficient of the kernel $K_{\text {Gauss }}$ compares the $i$ th coordinate of $\mathbf{x}$ to the $j$ th coordinate of $\mathbf{z}$, allowing a richer comparison between $\mathbf{x}$ and $\mathbf{z}$. For the sake of simplicity, we will call this kernel the Gaussian kernel in the remainder of the paper. Note that the Gaussian kernel depends on only one single hyperparameter $\gamma$. It gives rise to the following Gaussian OKVAR model. 
Definition 3 (Gaussian OKVAR)

$$
h_{\text {Gauss }}\left(\mathbf{x}_{t}\right)=\sum_{\ell=1}^{N} K_{\text {Gauss }}\left(\mathbf{x}_{t}, \mathbf{x}_{\ell}\right) \mathbf{c}_{\ell}
$$

An interesting feature of the Gaussian kernel-based OKVAR model is that each coordinate $i$ of the vector model $h_{\text {Gauss }}\left(\mathbf{x}_{t}\right)^{i}$ can be expressed as a linear combination of nonlinear functions of variables $j=1, \ldots, d: h_{\text {Gauss }}\left(\mathbf{x}_{t}\right)^{i}=\sum_{\ell} \sum_{j} \exp \left(-\gamma\left(x_{t}^{i}-x_{\ell}^{j}\right)^{2}\right) c_{\ell}^{j}$.

Decomposable kernels are another class of kernels, first defined by Micchelli and Pontil (2005) to address multi-task regression problems and structured classification. When based on Gaussian kernels, they are defined as follows:

Definition 4 (Decomposable (Gaussian) kernel) Let $B$ be a positive semi-definite matrix of size $d \times d$.

$$
\forall(\mathbf{x}, \mathbf{z}) \in \mathbb{R}^{d} \times \mathbb{R}^{d}, K_{\text {dec }}(\mathbf{x}, \mathbf{z})=k_{\text {Gauss }}(\mathbf{x}, \mathbf{z}) B
$$

In this kernel, $B$ is related to the structure underlying the outputs: $B$ imposes dependence amongst selected outputs. This kernel was shown to be universal by Caponnetto et al. (2008), e.g. the induced RKHS is a family of universal approximators. The decomposable Gaussian OKVAR model is then defined as follows:

Definition 5 (Decomposable Gaussian OKVAR)

$$
h_{d e c}\left(\mathbf{x}_{t}\right)=\sum_{\ell=1}^{N} \exp \left(-\gamma\left\|\mathbf{x}_{t}-\mathbf{x}_{\ell}\right\|^{2}\right) B \mathbf{c}_{\ell}
$$

Let now $K_{d e c}$ be a decomposable Gaussian kernel with scalar parameter $\gamma_{1}$ and matrix parameter $B$ and $K_{\text {Gauss }}$ be a Gaussian kernel with scalar parameter $\gamma_{2}$. As proposed in Lim et al. (2013), we combine the Gaussian kernel and the decomposable kernel with the Hadamard product to get a kernel that involves nonlinear functions of single coordinates of the input vectors, while imposing some structure to the kernel through a positive semi-definite matrix $B$. The resulting kernel is called the Hadamard kernel.

Definition 6 (Hadamard kernel)

$$
\forall(\mathbf{x}, \mathbf{z}) \in \mathbb{R}^{d} \times \mathbb{R}^{d}, K_{\text {Hadamard }}(\mathbf{x}, \mathbf{z})=K_{\text {dec }}(\mathbf{x}, \mathbf{z}) \circ K_{\text {Gauss }}(\mathbf{x}, \mathbf{z})
$$

where $\circ$ denotes the Hadamard product for matrices.

The resulting kernel $K_{\text {Hadamard }}$ possesses the kernel property, i.e:

Proposition 1 The kernel defined by (11) is a matrix-valued kernel.

Proof A Hadamard product of two matrix-valued kernels is a matrix-valued kernel [proposition 4 in Caponnetto et al. (2008)].

The Hadamard OKVAR model has the following form:

\section{Definition 7 (Hadamard OKVAR)}

$$
h_{\text {Hadamard }}\left(\mathbf{x}_{t}\right)=\sum_{\ell=1}^{N} \exp \left(-\gamma_{1}\left\|\mathbf{x}_{t}-\mathbf{x}_{\ell}\right\|^{2}\right) B \circ K_{\text {Gauss }}\left(\mathbf{x}_{t}, \mathbf{x}_{\ell}\right) \mathbf{c}_{\ell}
$$




\subsection{Jacobians of the OKVAR models}

As mentioned in the introduction, the network structure will be inferred by the empirical mean of the instantaneous Jacobian matrices $\hat{J}(h)(t)$ of $h$ over observed time-points. For any matrix-valued kernel-based model $h$, at any given time point $t$, for a given target state variable $i$, we have:

$$
\forall j \in\{1, \ldots, d\}, \hat{J}(h)_{i j}(t)=\frac{\partial h\left(\mathbf{x}_{t} ; \mathbf{x}_{1}, \ldots, \mathbf{x}_{t}, \ldots, \mathbf{x}_{N}\right)^{i}}{\partial x_{t}^{j}}=\sum_{\ell=1}^{N} \frac{\partial\left(K\left(\mathbf{x}_{t}, \mathbf{x}_{\ell}\right) \mathbf{c}_{\ell}\right)^{i}}{\partial x_{t}^{j}}
$$

Hence, each component of $h$ should be a function of the state variables in such a way that the coefficients of the Jacobian reflect the dependence of the output component on some of the state variables. Due to our assumption of nonlinear dynamics of the underlying system, the kernel should contain nonlinear functions of the state variables. Moreover, a relevant matrix-valued kernel-based model should allow the sparsity of the Jacobian to be controlled through the values of its parameters. The kernels proposed previously in Sect. 3.3 give rise to the following expressions for instantaneous Jacobian.

Gaussian OKVAR. The $(i, j)$ th entry of the Jacobian at time $t$ for the Gaussian-OKVAR model (8) is:

$$
\hat{J}\left(h_{\text {Gauss }}\right)_{i j}(t)=2 \gamma\left(x_{t}^{i}-x_{t}^{j}\right) \exp \left(-\gamma\left(x_{t}^{i}-x_{t}^{j}\right)^{2}\right) c_{t}^{j},
$$

which implies that the $c_{t}^{j}$ 's have the same impact, no matter what the target variable $i$ is. As a consequence, it becomes impossible to control those parameters for network inference purposes.

Decomposable OKVAR. If we now consider the decomposable model, $h_{\text {dec }}$, defined in (10), the corresponding $(i, j)$ th term of the Jacobian is given by:

$$
\hat{J}\left(h_{d e c}\right)_{i j}(t)=\sum_{\ell=1}^{N} \frac{\partial \exp \left(-\gamma\left\|\mathbf{x}_{t}-\mathbf{x}_{\ell}\right\|^{2}\right)}{\partial x_{t}^{j}}\left(B \mathbf{c}_{\ell}\right)^{i}
$$

which implies that the nonlinear term involved in the matrix-valued kernel does not differ from one pair $(i, j)$ to another. Then, it is impossible to control specific values of the Jacobian matrix using $B$ or the $\mathbf{c}_{\ell}$ 's.

Hadamard OKVAR. Finally, we obtain a richer class of Jacobians, more suitable for the inference task at hand, if we use Hadamard OKVAR defined in (12) for which the entries of its Jacobian at time $t \hat{J}\left(h_{\text {Hadamard }}\right)_{i j}(t)=\hat{J}_{i j}(t)$ are given by:

$$
\begin{aligned}
\hat{J}_{i j}(t)= & \sum_{\ell=1}^{N} \frac{\partial \exp \left(-\gamma_{1}\left\|\mathbf{x}_{t}-\mathbf{x}_{\ell}\right\|^{2}\right)}{\partial x_{t}^{j}}\left(B \circ K_{\text {Gauss }}\left(\mathbf{x}_{t}, \mathbf{x}_{\ell}\right) \mathbf{c}_{\ell}\right)^{i}+\exp \left(-\gamma_{1}\left\|\mathbf{x}_{t}-\mathbf{x}_{\ell}\right\|^{2}\right) \\
& \times \frac{\partial\left(B \circ K_{\text {Gauss }}\left(\mathbf{x}_{t}, \mathbf{x}_{\ell}\right) \mathbf{c}_{\ell}\right)^{i}}{\partial x_{t}^{j}}
\end{aligned}
$$


which after some calculations reduces to:

$$
\begin{aligned}
\hat{J}_{i j}(t)= & 2 \gamma_{2} b_{i j}\left(x_{t}^{i}-x_{t}^{j}\right) \exp \left(-\gamma_{2}\left(x_{t}^{i}-x_{t}^{j}\right)^{2}\right) c_{t}^{j} \\
& -2 \gamma_{1} \sum_{\ell \neq t} \exp \left(-\gamma_{1}\left\|\mathbf{x}_{t}-\mathbf{x}_{\ell}\right\|^{2}\right)\left(x_{t}^{j}-x_{\ell}^{j}\right) \sum_{p=1}^{d} b_{i p} \exp \left(-\gamma_{2}\left(x_{t}^{i}-x_{\ell}^{p}\right)^{2}\right) c_{\ell}^{p}
\end{aligned}
$$

The obtained expression exhibits some interesting characteristics: if we choose $\gamma_{1}$ very close to 0 (for $\gamma_{1}=0, k_{\text {Gauss }}$ is no more a kernel), then $k_{\text {Gauss }}\left(\mathbf{x}_{t}, \mathbf{x}_{\ell}\right)$ is close to one for any pair $\left(\mathbf{x}_{t}, \mathbf{x}_{\ell}\right)$ and the second term in (15) is approximatively 0 . Then, the value of the Jacobian for variables $(i, j)$ is controlled by the value of $b_{i j}$, the $(i, j)$ th entry of $B$ : hence, $B$ is capable of imposing structure in the model. For example, for a given pair of variables $(i, j)$, if $b_{i j}=0$, then irrespective of the values of $c_{\ell}^{j}, \ell=1, \ldots, N$, the corresponding Jacobian coefficient will be approximately zero as well since $\gamma_{1}$ is very close to 0 ; i.e variable $j$ does not influence variable $i$. Conversely, a non-zero coefficient $b_{i j}$ does not reflect influence from $j$ to $i$ since the $\mathbf{c}_{\ell}$ parameters can still set the corresponding coefficient in the Jacobian to zero. Thus, the parameter $B$ captures some of the structure of the underlying network, together with $C$. Note that the vectors $\mathbf{c}_{\ell}$ 's and the cross-difference between coordinates in Eq. (15) allow us to have non-symmetric Jacobian matrices, suitable for reconstructing directed graphs.

\section{Learning OKVAR with proximal gradient algorithms}

\subsection{Learning $C$ for fixed kernel}

In some applications, kernel $K$ may be already specified. For instance, the transformable Gaussian kernel depends on a parameter $\gamma_{2}$ that might be preset. For a decomposable or a Hadamard kernel, the matrix $B$ may be provided a priori. Thus, learning the resulting OKVAR model boils down to learning the matrix of model parameters $C$. We denote the model $h_{C}$ to highlight that dependence. To estimate $C$, we employ the following general regularized loss function:

$$
\mathscr{L}(C)=\sum_{t=1}^{N}\left\|h_{C}\left(\mathbf{x}_{t}\right)-\mathbf{x}_{t+1}\right\|^{2}+\lambda_{h}\left\|h_{C}\right\|_{\mathscr{H}}^{2}+\Omega(C)
$$

The squared norm $\left\|h_{C}\right\|_{\mathscr{H}}^{2}=\sum_{i, j=1}^{N} \mathbf{c}_{i}^{T} K\left(\mathbf{x}_{i}, \mathbf{x}_{j}\right) \mathbf{c}_{j}$ plays the role of a weighted $\ell_{2}$ norm on $C$. When $\Omega(C)=0$, minimizing (16) corresponds to solving the kernel ridge regression problem. In this case, $C$ can be computed in closed-form:

$$
\mathbf{c}=\left(\mathbf{K}+\lambda_{h} I d\right)^{-1} \mathbf{x}_{2: N+1}
$$

where $\mathbf{c}$ is the vectorized form of matrix $C, \mathbf{K}=\left(K\left(\mathbf{x}_{\ell}, \mathbf{x}_{t}\right)\right)_{\ell, t} \in \mathscr{M}^{N d}$ is the blockGram matrix computed on pairs of data $\left(\mathbf{x}_{\ell}, \mathbf{x}_{t}\right), \ell, t=1 \ldots N$ and $\mathbf{x}_{2: N+1} \in \mathbb{R}^{N d}$ is the concatenated vector of data $\mathbf{x}_{2}, \ldots, \mathbf{x}_{N+1}$. However this solution is usually not sparse. In order to control the sparsity of the model, necessary for obtaining a sparse Jacobian, one may introduce an $\ell_{1}$-norm constraint on $C$, that is $\Omega_{1}(C)=\lambda_{C}\|C\|_{1}$ where $\|\cdot\|_{1}$ denotes both the $\ell_{1}$ norm of a vector and that of the vectorized form of a matrix. Then the loss function becomes analogous to the one used in elastic-net type regularized models in the scalar case. 
In nonparametric approaches, one key issue is to control model complexity. A way to achieve it is to make use of a few parameters $\mathbf{c}_{\ell}$, which implies that only a few data points are involved in the model. In analogy to Support Vector Machines, we denote by Support Vectors data corresponding to a null vector $\mathbf{c}_{\ell}$. Regularizing by the $\ell_{1}$-norm cannot set a whole vector $\mathbf{c}_{\ell}$ to zero, i.e. exhibit support vectors. To achieve this goal, a structured sparsity strategy is more appropriate by considering the columns of $C$, i.e. vectors $\mathbf{c}_{\ell}$ 's, as a partition of the matrix coefficients. Such a constraint $\Omega_{\text {struct }}$ may take the following form:

$$
\Omega_{\text {struct }}(C)=\lambda_{C} \sum_{\ell=1}^{N} w_{\ell}\left\|\mathbf{c}_{\ell}\right\|_{2}
$$

As it is defined in (18), $\Omega_{\text {struct }}$ is the so-called mixed $\ell_{1} / \ell_{2}$-norm. First used in Yuan and Lin (2006) for the group lasso formulation, this norm exhibits some interesting features : it behaves like an $\ell_{1}$-norm on each vector $\mathbf{c}_{\ell}$ while within each vector $\mathbf{c}_{\ell}$, the coefficients are subject to an $\ell_{2}$-norm constraint. Note that $w_{\ell}$ 's are positive weights whose values depend on the application.

Hence, (16) is a convex loss function that is a sum of two terms: $f_{C}$ which is differentiable with respect to $C$ and $g_{C}$ which is non-smooth, but nevertheless convex and subdifferentiable with respect to $C$ :

$$
\mathscr{L}(C)=\underbrace{\sum_{t=1}^{N}\left\|\sum_{\ell=1}^{N} K\left(\mathbf{x}_{t}, \mathbf{x}_{\ell}\right) \mathbf{c}_{\ell}-\mathbf{x}_{t+1}\right\|^{2}+\lambda_{h} \sum_{t, \ell=1}^{N} \mathbf{c}_{t}^{T} K\left(\mathbf{x}_{t}, \mathbf{x}_{\ell}\right) \mathbf{c}_{\ell}}_{f_{C}(C)}+\underbrace{\Omega(C)}_{g_{C}(C)}
$$

This leads us to employ a proximal gradient algorithm, which is appropriate for solving this problem (see Combettes and Pesquet (2011) and references therein). Its steps are outlined in Algorithm 1 which relies on the following:

- $L_{C}$ is a Lipschitz constant of $\nabla_{C} f_{C}$, the derivative of $f_{C}$ for variable $C$

- For $s>0$, the proximal operator of a function $g$ applied to some $\mathbf{v} \in \mathbb{R}^{N d}$ is given by: $\operatorname{prox}_{s}(g)(\mathbf{v})=\operatorname{argmin}_{\mathbf{u}}\left\{g(\mathbf{u})+\frac{1}{2 s}\|\mathbf{u}-\mathbf{v}\|^{2}\right\}$

- Intermediary variables $t^{(m)}$ and $\mathbf{y}^{(m)}$ in Step 2 and Step 3 respectively are introduced to accelerate the proximal gradient method (Beck and Teboulle 2010).

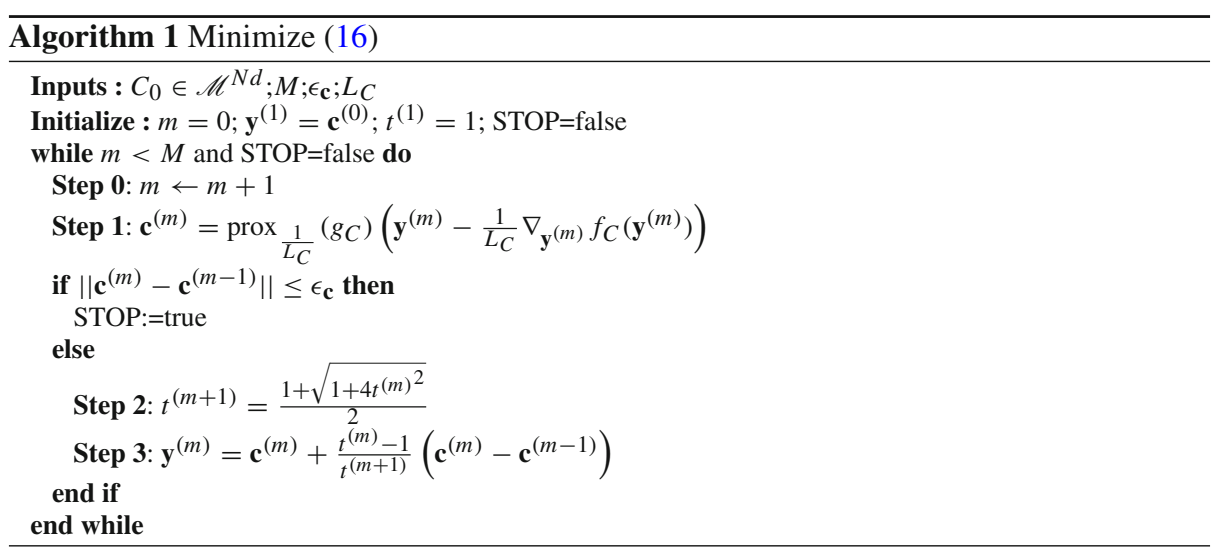


Let $\mathscr{G}$ be a partition of the indices of $\mathbf{v} \in \mathbb{R}^{N d}$, for a given subset of indices $I \in \mathscr{G}$, the proximal operator of $\Omega_{1}$ or $\Omega_{\text {struct }}$ is the elementwise shrinkage or soft-thresholding operator $\mathscr{T}_{s}: \mathbb{R}^{N d} \rightarrow \mathbb{R}^{N d}:$

$$
\mathscr{T}_{S}(\mathbf{v})_{I}=\left(1-\frac{s}{\left\|\mathbf{v}^{I}\right\|_{2}}\right)_{+} \mathbf{v}^{I}
$$

where $\mathbf{v}^{I} \in \mathbb{R}^{|I|}$ denotes the coefficients of $\mathbf{v}$ indexed by $I$. Then, the proximal gradient term in the $m^{\text {th }}$ iteration is given by:

$$
\operatorname{prox}_{\frac{1}{L_{C}}}\left(g_{C}\right)\left(\mathbf{y}^{(m)}-\frac{1}{L_{C}} \nabla_{\mathbf{y}^{(m)}} f_{C}\left(\mathbf{y}^{(m)}\right)\right)_{I_{\ell}}=\mathscr{T}_{S_{\ell}}\left(\mathbf{y}^{(m)}-\frac{1}{L_{C}} \nabla_{\mathbf{y}^{(m)}} f_{C}\left(\mathbf{y}^{(m)}\right)\right)_{I_{\ell}}
$$

For $g_{C}=\Omega_{\text {struct }}, s_{\ell}=\frac{\lambda_{C} w_{\ell}}{L_{C}}$ and $I_{\ell}, \ell=1, \ldots, N$ is the subset of indices corresponding to the $\ell$-th column of $C$, while for $g_{C}=\Omega_{1}, s_{\ell}=\frac{\lambda_{C}}{L_{C}}$ and $I_{\ell}, \ell=1, \ldots, N d$ is a singleton corresponding to a single entry of $C$.

We also need to calculate $L_{C}$ a Lipschitz constant of $\nabla_{C} f$. We can notice that $f_{C}(C)$ can be rewritten as:

$$
f_{C}(C)=\left\|\mathbf{K} \mathbf{c}-\mathbf{x}_{2: N+1}\right\|^{2}+\lambda_{h} \mathbf{c}^{T} \mathbf{K} \mathbf{c}
$$

Hence,

$$
\nabla_{C} f_{C}(C)=2 \mathbf{K}\left(\left[\mathbf{K}+\lambda_{h} I d\right] \mathbf{c}-\mathbf{x}_{2: N+1}\right)
$$

Using some algebra, we can establish that:

For $\mathbf{c}_{1}, \mathbf{c}_{2} \in \mathbb{R}^{N d}$,

$$
\left\|\nabla_{C} f_{C}\left(\mathbf{c}_{1}\right)-\nabla_{C} f_{C}\left(\mathbf{c}_{2}\right)\right\| \leq \underbrace{2 \rho\left(\mathbf{K}^{2}+\lambda_{h} \mathbf{K}\right)}_{L_{C}}\left\|\mathbf{c}_{1}-\mathbf{c}_{2}\right\|
$$

where $\rho\left(\mathbf{K}^{2}+\lambda_{h} \mathbf{K}\right)$, is the largest eigenvalue of $\mathbf{K}^{2}+\lambda_{h} \mathbf{K}$.

Remark It is of interest to notice that Algorithm 1 is very general and may be used as long as the loss function can be split into two convex terms, one of which is differentiable.

\subsection{Learning $C$ and the kernel}

Other applications require learning kernel $K$. For instance, in order to tackle the network inference task, one may choose a Gaussian decomposable kernel $K_{d e c}$ or a Hadamard kernel $K_{\text {Hadamard }}$. When bandwidth parameters $\gamma_{1}$ and $\gamma_{2}$ are preset, the only kernel hyperparameter to be learned is the positive semi-definite matrix $B$ that imposes structure in the model. This leads to the more involved task of simultaneously learning the matrix of the model parameters $C$, as well as $B$. Thus, we aim to minimize the following loss function for the two models $h_{\text {dec }}$ and $h_{\text {Hadamard }}$ :

$$
\mathscr{L}(B, C)=\sum_{t=1}^{N}\left\|h_{B, C}\left(\mathbf{x}_{t}\right)-\mathbf{x}_{t+1}\right\|^{2}+\lambda_{h}\left\|h_{B, C}\right\|_{\mathscr{H}}^{2}+\Omega(C)+\Omega(B)
$$

with $\Omega(C)$ a sparsity-inducing norm $\left(\Omega_{1}\right.$ or $\left.\Omega_{\text {struct }}\right), \Omega(B)=\lambda_{B}\|B\|_{1}$ and subject to the constraint that $B \in \mathscr{S}_{d}^{+}$where $\mathscr{S}_{d}^{+}$denotes the cone of positive semi-definite matrices of size $d \times d$. For fixed $\hat{C}$, the squared norm of $h_{B, \hat{C}}$ imposes a smoothing constraint on $B$, while the $\ell_{1}$ norm of $B$ aids in controlling the sparsity of the model and its Jacobian. 
Further, for fixed $\hat{B}$, the loss function $\mathscr{L}(\hat{B}, C)$ is convex in $C$ and conversely, for fixed $C, \mathscr{L}(B, \hat{C})$ is convex in $B$. We propose an alternating optimization scheme to minimize the overall loss $\mathscr{L}(B, C)$. Since both loss functions $\mathscr{L}(\hat{B}, C)$ and $\mathscr{L}(B, \hat{C})$ involve a sum of two terms, one being differentiable and the other being sub-differentiable, we employ proximal gradient algorithms to achieve the minimization.

For fixed $\hat{B}$, the loss function becomes:

$$
\mathscr{L}(\hat{B}, C)=\sum_{t=1}^{N}\left\|h_{\hat{B}, C}\left(\mathbf{x}_{t}\right)-\mathbf{x}_{t+1}\right\|^{2}+\lambda_{h}\left\|h_{\hat{B}, C}\right\|_{\mathscr{H}}^{2}+\Omega(C),
$$

while for given $\hat{C}$, it is given by:

$$
\mathscr{L}(B, \hat{C})=\sum_{t=1}^{N}\left\|h_{B, \hat{C}}\left(\mathbf{x}_{t}\right)-\mathbf{x}_{t+1}\right\|^{2}+\lambda_{h}\left\|h_{B, \hat{C}}\right\|_{\mathscr{H}}^{2}+\lambda_{B}\|B\|_{1}
$$

In summary, the general form of the algorithm is given in Algorithm 2.

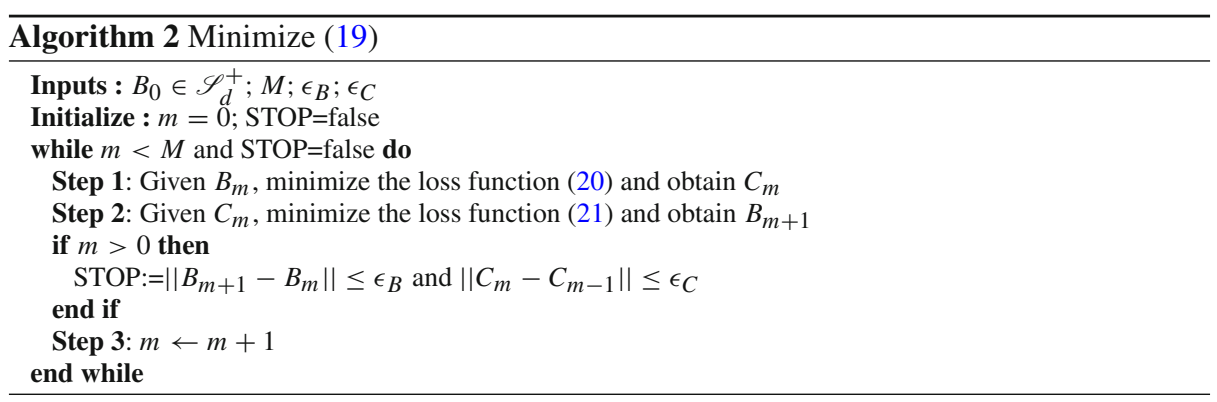

At iteration $m, B_{m}$ is fixed, and thus kernel $K$ is defined. Hence, estimation of $C_{m}$ in Step 1 boils down to applying Algorithm 1 to minimize (20).

\subsubsection{Learning the matrix $B$ for fixed $C$}

For given parameter matrix $C$, the loss function $\mathscr{L}(B, \hat{C})$ is minimized subject to the constraint that $B$ is positive semi-definite.

$$
\mathscr{L}(B, \hat{C})=\underbrace{\sum_{t=1}^{N}\left\|h_{B, \hat{C}}\left(\mathbf{x}_{t}\right)-\mathbf{x}_{t+1}\right\|^{2}+\lambda_{h}\left\|h_{B, \hat{C}}\right\|_{\mathscr{H}}^{2}}_{f_{B}(B)}+\underbrace{\lambda_{B}\|B\|_{1}}_{g_{B, 1}(B)}+\underbrace{1_{\mathscr{S}_{d}^{+}}(B)}_{g_{B, 2}(B)}
$$

where $1_{\mathscr{S}_{d}^{+}}$denotes the indicator function : $1_{\mathscr{S}_{d}^{+}}(B)=0$ if $B \in \mathscr{S}_{d}^{+},+\infty$ otherwise.

Note that $f_{B}$ is differentiable with respect to $B$, while both $g_{1, B}$ and $g_{2, B}$ are non-smooth, but convex and sub-differentiable with respect to $B$. When there is more than one non-smooth function involved in the loss function to minimize, we cannot use the same proximal gradient algorithm as delineated in Algorithm 1. We decide to adopt a strategy proposed by Raguet et al. (2011), where the authors generalize the classic forward-backward splitting algorithm to the case of an arbitrary number of non-smooth functions. The method has recently proven successful for the estimation of matrices with sparsity and low rank constraints (Richard et al. 2012). Our algorithm is presented below. 


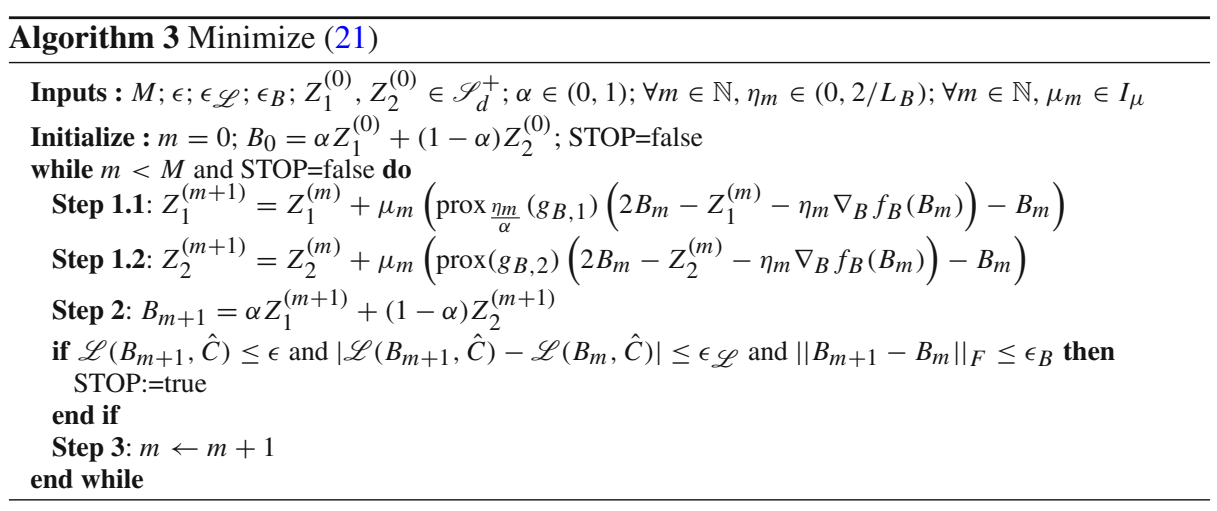

Two proximal operators need to be computed. The proximal operator of $g_{1, B}$ is the soft-thresholding operator while the proximal operator corresponding to the indicator function $1_{\mathscr{S}_{d}^{+}}$is the projection onto the cone of positive semidefinite matrices: for $Q \in$ $\mathscr{S}_{d}$, prox $\left(g_{B, 2}\right)(Q)=\operatorname{argmin}_{B \in \mathscr{S}_{d}^{+}}\|B-Q\|_{F}$. Sequence $\left(B_{m}\right)$ is guaranteed to converge under the following assumptions [Theorem 2.1 in Raguet et al. (2011)]:

Set $\overline{\lim } \eta_{m}=\bar{\eta}$,

(A) (i) $0<\underline{\lim } \mu_{m} \leq \varlimsup \lim \mu_{m}<\min \left(\frac{3}{2}, \frac{1+2 /\left(L_{B} \bar{\eta}\right)}{2}\right)$

(ii) $\sum_{m=0}^{+\infty}\left\|u_{2, m}\right\|<+\infty$, and for $i \in\{1,2\}, \sum_{m=0}^{+\infty}\left\|u_{1, m, i}\right\|<+\infty$

(B) (i) $0<\underline{\lim } \eta_{m} \leq \bar{\eta}<\frac{2}{L_{B}}$

(ii) $I_{\mu}=(0,1]$

where for $i \in\{1,2\}, u_{1, m, i}$ denotes the error at iteration $m$ when computing the proximal operator $\operatorname{prox}\left(g_{B, i}\right)$ and $u_{2, m}$ is the error when applying $\nabla_{B} f_{B}$ to its argument. These conditions are easily met by appropriate selection of $\alpha, \eta_{m}$ and $\mu_{m}$ as we do in the next section.

\section{Results}

\subsection{Implementation}

The performance of the developed OKVAR model family and the proposed optimization algorithms were assessed on two tracks: using simulated data from a biological system (DREAM3 challenge data set) and real climate data (Climate data set). These algorithms include a number of tuning parameters. Specifically, in Algorithm 3, we set $Z_{1}^{(0)}=Z_{2}^{(0)}=$ $B_{0} \in \mathscr{S}_{d}^{+}, \alpha=0.5$ and $\mu_{m}=1$. Parameters of the kernels were also fixed a priori: specifically, parameter $\gamma$ was set to 0.2 for a transformable Gaussian kernel and to 0.1 for a decomposable Gaussian kernel. In the case of a Hadamard kernel, two parameters need to be chosen: parameter $\gamma_{2}$ of the transformable Gaussian kernel remains unchanged $\left(\gamma_{2}=0.2\right)$. On the other hand, as discussed in Sect. 3.4, parameter $\gamma_{1}$ of the decomposable Gaussian kernel is fixed to a low value $\left(\gamma_{1}=10^{-5}\right)$ since it does not play a key role in the network inference task. 


\subsection{DREAM3 dataset}

We start our investigation by considering data sets obtained from the DREAM3 challenge (Prill et al. 2010). DREAM stands for Dialogue for Reverse Engineering Assessments and Methods (http://wiki.c2b2.columbia.edu/dream/index.php/The_DREAM_Project) and is a scientific consortium that organizes challenges in computational biology and especially for gene regulatory network inference. In a gene regulatory network, a gene $i$ is said to regulate another gene $j$ if the expression of gene $i$ at time $t$ influences the expression of gene $j$ at time $t+1$. The DREAM3 project provides realistic simulated data for several networks corresponding to different organisms (e.g. E. coli, Yeast, etc.) of different sizes and topological complexity. We focus here on size-10, size-50 and size-100 networks generated for the DREAM3 in silico challenges. Each of these networks corresponds to a subgraph of the currently accepted $E$. coli and $S$. cerevisiae gene regulatory networks and exhibits varying patterns of sparsity and topological structure. They are referred to as E1, E2, Y1, Y2 and Y3 with an indication of their size. The data were generated by imbuing the networks with dynamics from a thermodynamic model of gene expression and Gaussian noise. Specifically, 4, 23 and 46 time series consisting of 21 points were available respectively for size-10, size-50 and size-100 networks. We generated additional time series and extended their lengths up to 50 time points to study the behavior of the algorithm in various conditions. For that purpose, we used the same tool that generated the previously obtained time series, an open-source software called GeneNetWeaver (Schaffter et al. 2011), the generator that provided the DREAM3 competition with the network inference challenges.

In all the conducted experiments, we assess the performance of our model using the area under the ROC curve (AUROC) and under the Precision-Recall curve (AUPR) for regulation ignoring the sign (positive vs negative influence). The interested reader may however refer to the Supplementary Material where we provide additional results regarding this particular feature. The selected values for the hyperparameters for the penalty components $\lambda_{h}, \lambda_{C}$ and $\lambda_{B}$ are displayed in Table 1. For the DREAM3 data sets we also show the best results obtained from other competing teams using only time course data. The challenge made available other data sets, including ones obtained from perturbation (knock-out/knock-down) experiments, as well as observing the organism in steady state, but these were not considered in the results shown in the ensuing tables.

Further, several time series may also be available, because of multiple related initial conditions and/or technical replicates. In this case, the procedure is repeated accordingly. Hence, the predictions of each run on each time series are combined to build a consensus network. Specifically, for each run, we compute a rank matrix as described in Sect. 2. Then the coefficients of these matrices are averaged and eventually thresholded to obtain a final estimate of the adjacency matrix.

Table 1 Selected hyperparameters of OKVAR for DREAM3 size-10 and size-100 data sets

\begin{tabular}{|c|c|c|c|c|c|c|c|c|c|c|}
\hline & \multicolumn{5}{|c|}{ Size-10 } & \multicolumn{5}{|c|}{ Size-100 } \\
\hline & E1 & E2 & Y1 & Y2 & Y3 & E1 & E2 & Y1 & Y2 & Y3 \\
\hline$\lambda_{h}$ & 1 & 1 & 1 & 1 & 1 & 10 & 1 & 1 & 1 & 1 \\
\hline$\lambda_{C}$ & 0.01 & 1 & 1 & 1 & 1 & 10 & 0.01 & 100 & 100 & 100 \\
\hline$\lambda_{B}$ & 0.1 & 1 & 10 & 0.01 & 0.01 & 10 & 1 & 1 & 1 & 1 \\
\hline
\end{tabular}


Table 2 Synthetic table of studied OKVAR models

\begin{tabular}{llllllll}
\hline \multicolumn{5}{l}{ OKVAR models } & & & \\
\cline { 2 - 8 } & $h_{\text {Gauss }}^{\text {Ridge }}$ & $h_{\text {Gauss }}^{\ell_{1}}$ & $h_{\text {Gauss }}^{\ell_{1} / \ell_{2}}$ & $h_{\text {dec }}^{\ell_{1}}$ & $h_{\text {dec }}^{\ell_{1} / \ell_{2}}$ & $h_{\text {Hadamard }}^{\ell_{1}}$ & $h_{\text {Hadamard }}^{\ell_{1} / \ell_{2}}$ \\
\hline Kernel & \multicolumn{2}{l}{ Transformable Gaussian } & \multicolumn{2}{l}{ Decomposable Gaussian } & Hadamard & \\
Loss & Eq. (16) & & & \multicolumn{2}{l}{ Eq. (19) } & & \\
$\Omega(C)$ & 0 & $\Omega_{1}$ & $\Omega_{\text {struct }}$ & $\Omega_{1}$ & $\Omega_{\text {struct }}$ & $\Omega_{1}$ & $\Omega_{\text {struct }}$ \\
\hline
\end{tabular}

Table 3 Consensus AUROC and AUPR (given in \%) for the DREAM3 size-10 networks using the DREAM3 original data sets (4 time series of 21 points)

\begin{tabular}{|c|c|c|c|c|c|c|c|c|c|c|}
\hline \multirow[t]{2}{*}{ OKVAR models } & \multicolumn{5}{|c|}{ AUROC } & \multicolumn{5}{|c|}{ AUPR } \\
\hline & E1 & E2 & Y1 & $\mathrm{Y} 2$ & Y3 & E1 & E2 & Y1 & $\mathrm{Y} 2$ & Y3 \\
\hline $\begin{array}{l}\text { Ridge } \\
\text { Gauss }\end{array}$ & 68.8 & 37.7 & 62.1 & 68.6 & 66.7 & 15.6 & 11.2 & 15.5 & 46.9 & 32.9 \\
\hline$h_{\text {Gauss }}^{\ell_{1}}$ & 69.3 & 38.0 & 61.9 & 69.3 & 66.7 & 15.7 & 11.3 & 15.2 & 47.4 & 32.8 \\
\hline$h_{\text {Gauss }}^{\ell_{1} / \ell_{2}}$ & 68.7 & 37.1 & 62.4 & 68.6 & 66.7 & 15.5 & 11.1 & 15.6 & 47.5 & 32.6 \\
\hline$h_{d e c}^{\ell_{1}}$ & 67.0 & 68.5 & 38.2 & 45.4 & 38.3 & 23.6 & 20.8 & 7.4 & 21.1 & 16.8 \\
\hline$h_{d e c}^{\ell_{1} / \ell_{2}}$ & 65.9 & 47.8 & 45.3 & 56.6 & 38.5 & 23.1 & 14.0 & 8.3 & 28.5 & 16.8 \\
\hline$h_{\text {Hadamard }}^{\ell_{1}}$ & 81.2 & 46.2 & 47.7 & 76.2 & 70.5 & 23.5 & 12.7 & 8.7 & 50.1 & 39.5 \\
\hline$h_{\text {Hadamard }}^{\ell_{1} / \ell_{2}}$ & 81.5 & 78.7 & 76.5 & 70.3 & 75.1 & 32.1 & 50.1 & 35.4 & 37.4 & 39.7 \\
\hline
\end{tabular}

Studied OKVAR models refer to Table 2 . The numbers in boldface are the maximum values of each column

\subsubsection{Comparison between OKVAR models}

Next, we propose to compare the OKVAR models outlined in Table 2 and investigate how relevant these models are regarding the network inference task.

For the mixed $\ell_{1} / \ell_{2}$-norm regularization, we need to define coefficients $w_{\ell}$. As the observed time-course data correspond to the response of a dynamical system to some given initial condition, $w_{\ell}$ should increase with $\ell$, meaning that we put more emphasis on the first time-points. We thus propose to define $w_{\ell}$ as follows : $w_{\ell}=1-\exp (-(\ell-1))$.

Table 3 shows that the transformable Gaussian and the decomposable Gaussian kernels can achieve good performance on selected DREAM3 size-10 datasets, although none of these two kernels alone can faithfully recover all of the networks, no matter the type of sparsityinducing norms employed. On the other hand, the Hadamard kernel-based model learnt with a mixed $\ell_{1} / \ell_{2}$-norm regularization term, consistently outranks other OKVAR models. On the whole, these results tend to corroborate the discussion presented in Sect. 3.4. In the remainder of the paper, we focus on Hadamard kernel-based models that will be referred to as OKVAR-Prox.

\subsubsection{Effects of hyperparameters, noise, sample size and network size}

Next, we study the impact of different combinations of parameters including the sample size of the dataset (number of time points), the number of time series, the noise level and hyperparameters $\lambda_{C}$ and $\lambda_{B}$. 


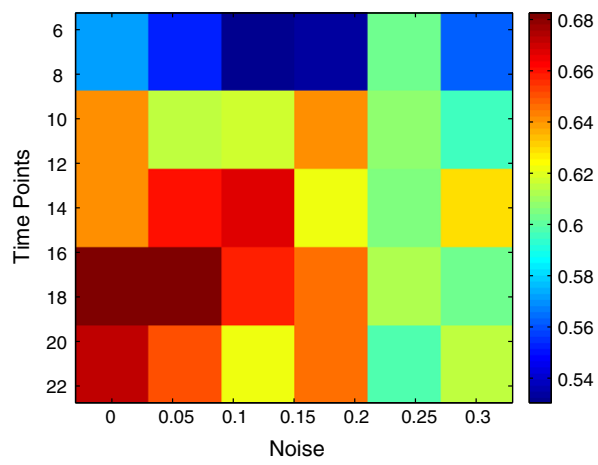

(a)

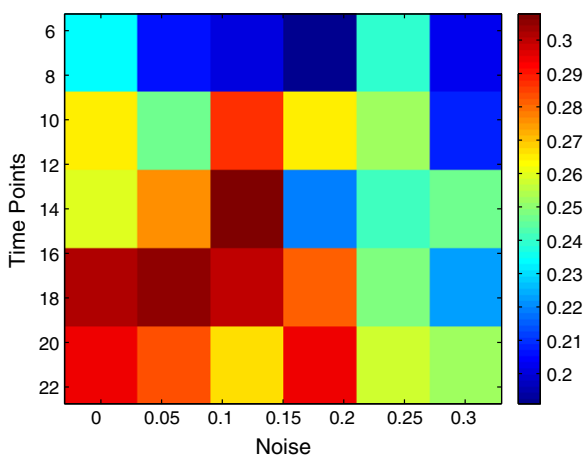

(b)

Fig. 1 Average consensus AUROC (a) and AUPR (b) on the 5 DREAM3 size-10 networks using the 4 DREAM3 original time series for different numbers of time points $(N=7,11,14,17$ and 21$)$ and different noise levels (standard deviations $\sigma=0,0.05,0.1,0.15,0.2$ and 0.3 )

Sample Size and Noise. We investigate the effect of both the noise and the length of time series on the algorithm's capability to reconstruct DREAM3 size-10 networks. For that purpose, we applied OKVAR-Prox on truncated time series consisting of $N=7,11,14,17$ and 21 time points, to which we added a zero-mean Gaussian noise with standard deviations $\sigma=0,0.05,0.1,0.15,0.2$ and 0.3 . As expected, performance gets worse with increasing levels of noise and decreasing number of time points (Fig. 1).

Sample Size for DREAM3 size-100 networks. We next study the way OKVAR is impacted in the less favorable case of large networks. The algorithm's performance for DREAM3 size-100 networks drops substantially with the very limited amount of data (21 time points) provided by the DREAM3 challenge, compared to size-10 networks' results. For this more involved task, we used the GeneNetWeaver software to extend the original time series with additional time points. Supplying the model with longer time series helps to slightly improve the network inference task, especially in terms of AUROC (Fig. 2). However as the dynamical systems under study (generated by DREAM3 consortium) are stable, a larger number of time points does not have a great impact here, especially on AUPR.

Network size and Noise. In order to assess the robustness of our method, we evaluate OKVARProx for different network sizes and various levels of noise and compare its performance with a LASSO-based approach (Table 4), which relies on an $\ell_{1}$-regularized linear least squares regression model of the form $x_{t+1}^{i}=\beta^{T} \mathbf{x}_{t}$, applied to each dimension (gene $i$ ). An edge is assigned for each nonzero $\beta$ coefficient.

Table 4 first indicates that both algorithms' performances deteriorate with increasing network sizes. Second, OKVAR-Prox clearly outranks the LASSO both in terms of AUROC and AUPR for any configuration of level noises and network sizes. Indeed, for a given size, OKVAR-Prox performs quite well in the presence of high levels of noise while the LASSO is strongly impacted by the noise, which advocates for OKVAR's robustness.

Network size and Number of time series. Figure 3a, b show that the network inference task is better performed when the algorithm is supplied with an increasing number of time series. The improvement is remarkably significant for size-10 networks. 


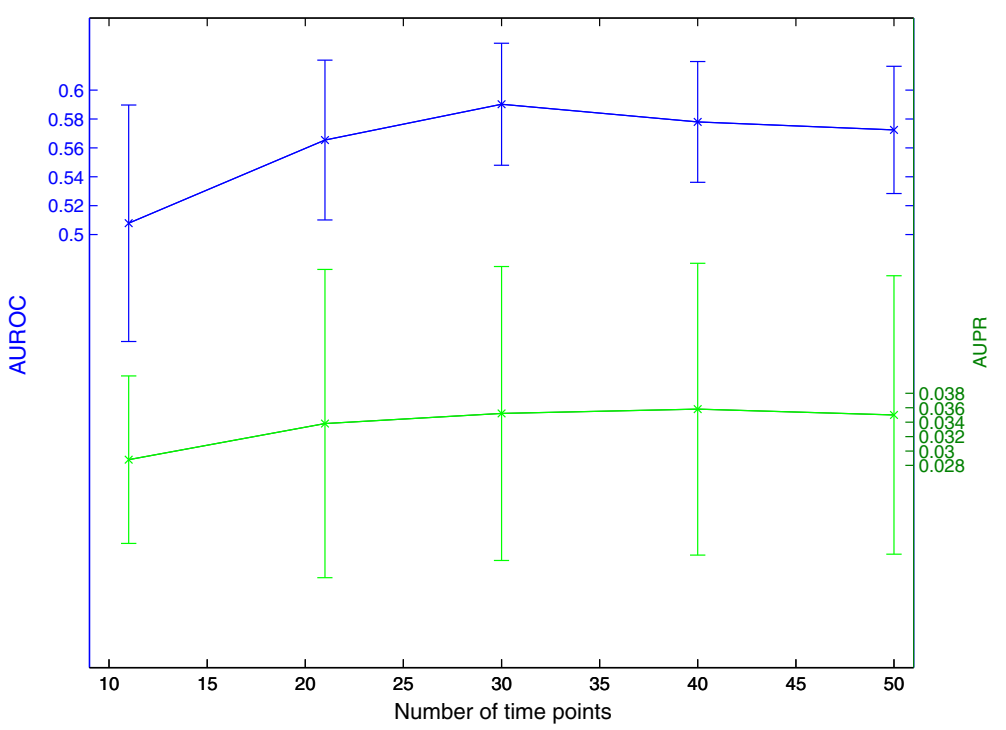

Fig. 2 Average consensus AUROC (blue line) and AUPR (green line) on the 5 DREAM3 size-100 networks using the data sets generated by GeneNetWeaver (20 time series) for different numbers of time points $(N=$ $11,21,30,40$ and 50)

Table 4 Consensus AUROC and AUPR (given in \%) obtained by OKVAR-Prox and the LASSO for DREAM3 E1 networks using the DREAM3 original data sets (4, 23 and 46 time series of 21 points for size-10, size-50, and size-100 E1 respectively) and different noise levels (standard deviations $\sigma=0,0.05,0.1,0.15,0.2$ and $0.3)$

\begin{tabular}{|c|c|c|c|c|c|c|c|c|c|c|c|c|}
\hline \multirow[b]{2}{*}{ Size-10 E1 } & \multicolumn{6}{|c|}{ AUROC } & \multicolumn{6}{|c|}{ AUPR } \\
\hline & & & & & & & & & & & & \\
\hline Noise $(\sigma)$ & 0 & 0.05 & 0.1 & 0.15 & 0.2 & 0.3 & 0 & 0.05 & 0.1 & 0.15 & 0.2 & 0.3 \\
\hline OKVAR-Prox & 81.5 & 67.9 & 72.0 & 75.2 & 72.4 & 74.4 & 32.1 & 20.7 & 21.2 & 24.3 & 22.7 & 21.4 \\
\hline LASSO & 69.5 & 64.2 & 61.7 & 43.0 & 50.7 & 48.8 & 17.0 & 13.9 & 17.8 & 9.0 & 10.1 & 10.7 \\
\hline \multicolumn{13}{|l|}{ Size-50 E1 } \\
\hline Noise $(\sigma)$ & 0 & 0.05 & 0.1 & 0.15 & 0.2 & 0.3 & 0 & 0.05 & 0.1 & 0.15 & 0.2 & 0.3 \\
\hline OKVAR-Prox & 66.4 & 67.3 & 68.6 & 69.2 & 69.8 & 70.9 & 4.1 & 4.3 & 5.0 & 5.9 & 6.6 & 6.9 \\
\hline LASSO & 52.8 & 54.5 & 46.0 & 54.9 & 45.9 & 50.7 & 2.9 & 2.8 & 2.1 & 3.5 & 2.2 & 2.6 \\
\hline \multicolumn{13}{|l|}{ Size-100 E1 } \\
\hline Noise $(\sigma)$ & 0 & 0.05 & 0.1 & 0.15 & 0.2 & 0.3 & 0 & 0.05 & 0.1 & 0.15 & 0.2 & 0.3 \\
\hline OKVAR-Prox & 65.4 & 56.1 & 56.5 & 56.4 & 57.2 & 58.0 & 4.6 & 1.7 & 1.7 & 1.7 & 1.7 & 1.8 \\
\hline LASSO & 52.2 & 54.3 & 53.3 & 47.2 & 51.3 & 51.5 & 1.4 & 1.6 & 1.4 & 1.2 & 1.3 & 1.3 \\
\hline
\end{tabular}

The numbers in boldface are the maximum values of each column

Hyperparameters. We also studied the impact of the hyperparameters. We notice that both AUROC and AUPR values are not that sensitive to small changes in the hyperparameters (Table 5), which strongly indicates that it is sufficient to pick them within a reasonable range of values. 

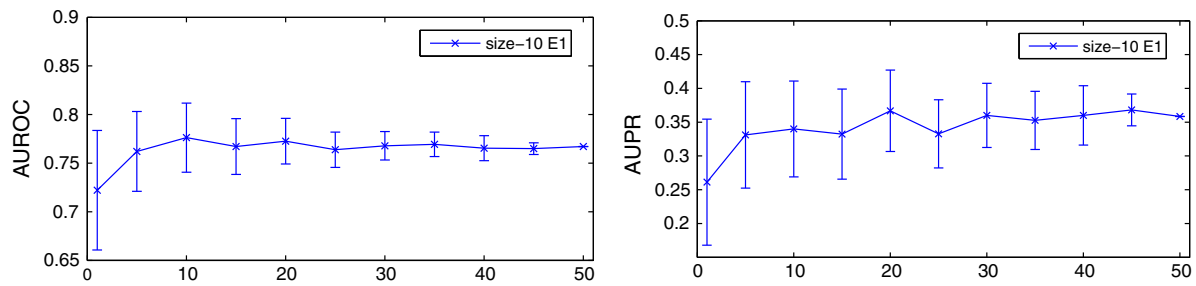

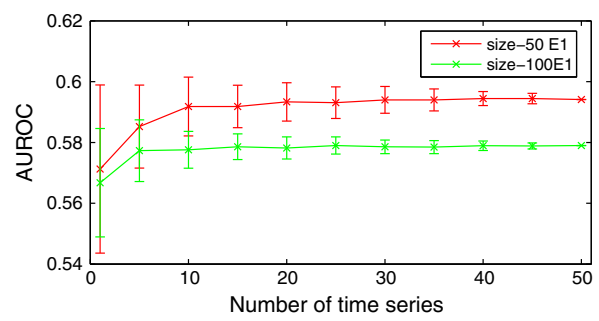

(a)

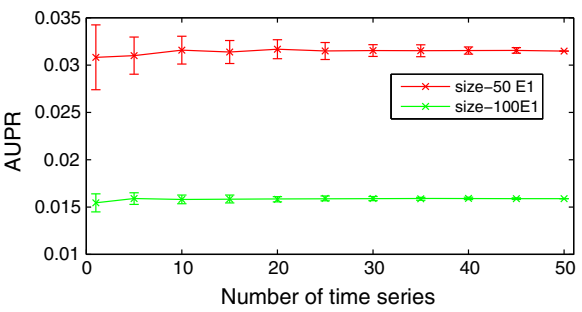

(b)

Fig. 3 Consensus AUROC (a) and AUPR (b) for size-10 (blue line), size-50 (red line) and size-100 (green line) DREAM3 E1 network using 1 up to 50 time series of 50 points respectively. Standard deviations (error bars) correspond to different combinations of time series

Table 5 Consensus AUROC and AUPR (given in \%) for the DREAM3 size-10 E1 network using the DREAM3 original data sets (4 time series of 21 points) for different combinations of hyperparameters $\left(\lambda_{C}, \lambda_{B}\right)$

\begin{tabular}{lllll}
\hline \multicolumn{5}{c}{$\lambda_{B}$} \\
\cline { 3 - 4 } & & $10^{-2}$ & $10^{-1}$ & 1 \\
\hline$\lambda_{C}$ & $10^{-2}$ & $79.1 / 31.5$ & $81.5 / 32.1$ & $73.5 / 21.2$ \\
& $10^{-1}$ & $79.7 / 36.5$ & $76.9 / 21.6$ & $66.7 / 25.9$ \\
& 1 & $78.1 / 25.9$ & $71.0 / 20.7$ & $61.4 / 16.0$ \\
\hline
\end{tabular}

$\lambda_{h}=1$

\subsubsection{Choice of penalty component}

In order to study the impact of the type of regularization employed, we assessed OKVAR's performance on the following three network tasks: size-10, size-50 and size-100 E1 networks.

Figure $4 \mathrm{a}-\mathrm{c}$ highlight that the choice of the regularization depends on the size of the problem. Specifically, a mixed $\ell_{1} / \ell_{2}$-norm regularization helps in the inference task when the ratio between the number of measurements and the network size is favorable, which is the case for DREAM3 size-10 networks while it remains unclear whether a mixed-norm or a pure $\ell_{1}$-norm penalty is more appropriate for large networks when very few data are available, e.g. DREAM3 size-50 and size-100 networks.

\subsubsection{Comparison with state-of-the-art methods}

The performance of the OKVAR approach for prediction of the network structure is assessed on DREAM3 size-10 and size-100 datasets.

Results are presented in Tables 6 and 7 for size- 10 and size- 100 data sets, respectively. The entries of these tables correspond to the following methods: (i) OKVAR + True B corresponds 


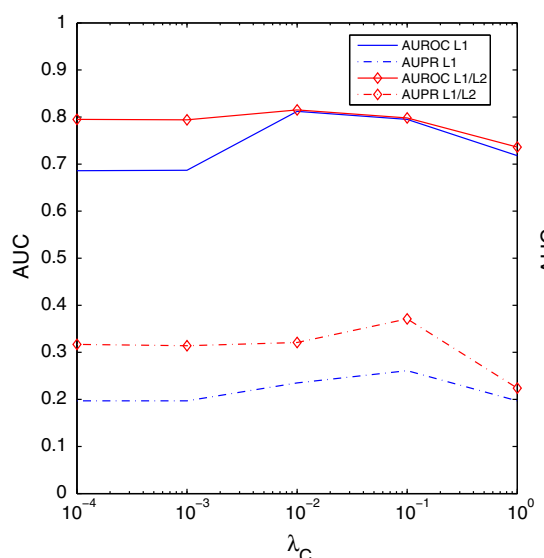

(a)

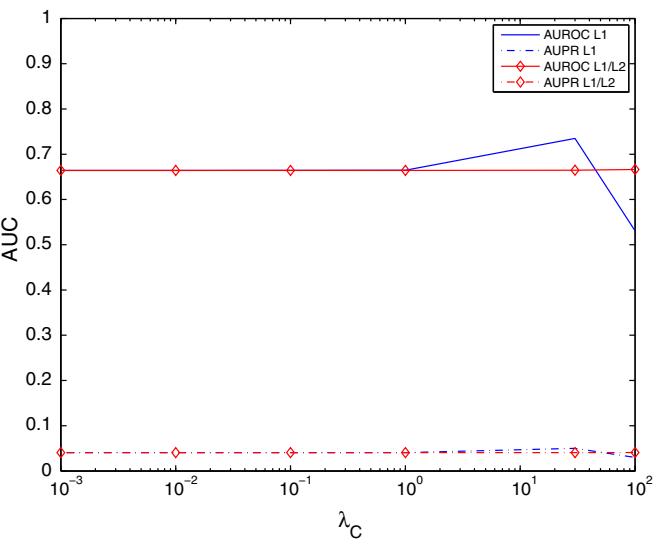

(b)

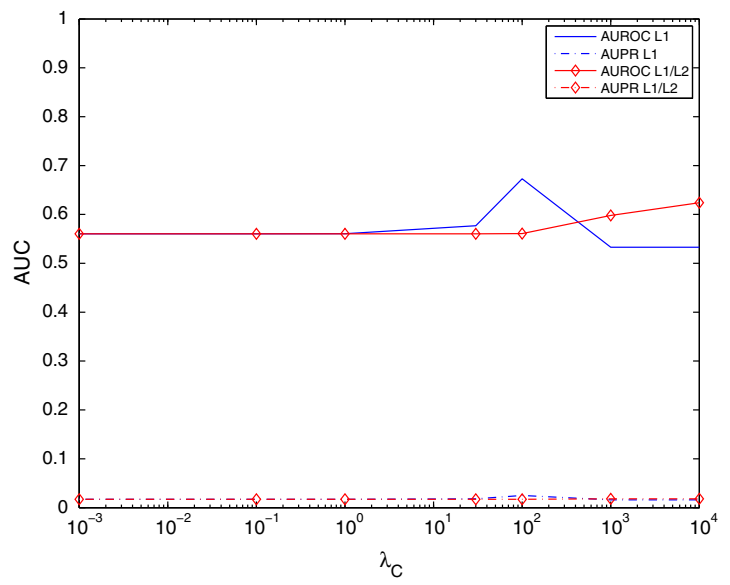

(c)

Fig. 4 Consensus AUROC and AUPR for a size-10, b size-50 and c size-100 DREAM3 E1 networks using the DREAM3 original data sets $(4,23$ and 46 time series of 21 points for size-10, size-50 and size-100 E1 respectively) for an $\ell_{1}$-norm (blue line) or a mixed $\ell_{1} / \ell_{2}$-norm (red line) regularization

to an OKVAR model with the true adjacency matrix given, and projected onto the positive semidefinite cone. The obtained results for this row provide an upper bound on the expected performance of the model. (ii) OKVAR-Prox was learnt using a mixed $\ell_{1} / \ell_{2}$-norm constraint on model parameters for size-10 datasets and an $\ell_{1}$-norm for size-100 datasets. (iii) The LASSO. (iv) G1DBN is an algorithm that performs Dynamic Bayesian Network inference using first-order conditional dependencies (Lèbre 2009). (v) GPODE is a structure inference method based on non-parametric Gaussian process modeling and parameter estimation of ordinary differential equations (Aijo and Lahdesmaki 2009). (vi, vii) Finally, the two last rows present the results from two competing teams that exhibited a very good performance based only on similar time-series data. Although there is no information on the structure of Team 236's algorithm, its authors responded to the post-competition DREAM3 survey stating that their method employs Bayesian models with an in-degree constraint (Prill et al. 
Table 6 Consensus AUROC and AUPR (given in \%) for OKVAR-Prox, LASSO, GPODE, G1DBN, Team 236 and Team 190 (DREAM3 competing teams) run on DREAM3 size-10 networks using the DREAM3 original data sets (4 time series of 21 points)

\begin{tabular}{|c|c|c|c|c|c|c|c|c|c|c|}
\hline \multirow[t]{2}{*}{ Size-10 } & \multicolumn{5}{|c|}{ AUROC } & \multicolumn{5}{|c|}{ AUPR } \\
\hline & E1 & E2 & Y1 & Y2 & Y3 & E1 & E2 & $\mathrm{Y} 1$ & $\mathrm{Y} 2$ & Y3 \\
\hline OKVAR + True $B$ & 96.2 & 86.9 & 89.2 & 75.6 & 86.6 & 75.2 & 67.7 & 47.3 & 52.3 & 58.6 \\
\hline OKVAR-Prox & 81.5 & 78.7 & 76.5 & 70.3 & 75.1 & 32.1 & 50.1 & 35.4 & 37.4 & 39.7 \\
\hline LASSO & 69.5 & 57.2 & 46.6 & 62.0 & 54.5 & 17.0 & 16.9 & 8.5 & 32.9 & 23.2 \\
\hline GPODE & 60.7 & 51.6 & 49.4 & 61.3 & 57.1 & 18.0 & 14.6 & 8.9 & 37.7 & 34.1 \\
\hline G1DBN & 63.4 & 77.4 & 60.9 & 50.3 & 62.4 & 16.5 & 36.4 & 11.6 & 23.2 & 26.3 \\
\hline Team 236 & 62.1 & 65.0 & 64.6 & 43.8 & 48.8 & 19.7 & 37.8 & 19.4 & 23.6 & 23.9 \\
\hline Team 190 & 57.3 & 51.5 & 63.1 & 57.7 & 60.3 & 15.2 & 18.1 & 16.7 & 37.1 & 37.3 \\
\hline
\end{tabular}

The numbers in boldface are the maximum values of each column

Table 7 Consensus AUROC and AUPR (given in \%) for OKVAR-Prox, LASSO, G1DBN, Team 236 (DREAM3 competing team) run on DREAM3 size-100 networks using the DREAM3 original data sets (46 time series of 21 points)

\begin{tabular}{|c|c|c|c|c|c|c|c|c|c|c|}
\hline \multirow[t]{2}{*}{ Size-100 } & \multicolumn{5}{|c|}{ AUROC } & \multicolumn{5}{|c|}{ AUPR } \\
\hline & E1 & E2 & Y1 & Y2 & Y3 & E1 & E2 & Y1 & $\mathrm{Y} 2$ & Y3 \\
\hline OKVAR + True $B$ & 96.2 & 97.1 & 95.8 & 90.6 & 89.7 & 43.2 & 51.6 & 27.9 & 40.7 & 36.4 \\
\hline OKVAR-Prox & 65.4 & 64.0 & 54.9 & 56.8 & $\mathbf{5 3 . 5}$ & 4.6 & 2.6 & 2.3 & 5.0 & 6.3 \\
\hline LASSO & 52.2 & 55.0 & 53.2 & 52.4 & 52.3 & 1.4 & 1.3 & 1.8 & 4.3 & 6.1 \\
\hline G1DBN & 53.4 & 55.8 & 47.0 & 58.1 & 43.4 & 1.6 & 6.3 & 2.2 & 4.6 & 4.4 \\
\hline Team 236 & 52.7 & 54.6 & 53.2 & 50.8 & 50.8 & 1.9 & 4.2 & 3.5 & 4.6 & 6.5 \\
\hline
\end{tabular}

The numbers in boldface are the maximum values of each column

2010). Team 190 (Table 6) reported in the same survey that their method is also Bayesian with a focus on nonlinear dynamics and local optimization.

The AUROC and AUPR values obtained for size-10 networks (Table 6) strongly indicate that OKVAR-Prox outperforms state-of-the-art models and the teams that exclusively used the same set of time series data in the DREAM3 competition, except for size-10 Y2 (nearly equivalent AUPR). In particular, we note that the OKVAR consensus runs exhibited excellent AUPR values compared to those obtained by other approaches.

A comparison of competing algorithms for size-100 networks (Table 7) shows that the OKVAR method again achieves superior AUROC results compared to Team 236, although it only lags behind by a slight margin for size-100 Y1 and Y3 in terms of AUPR. Team 236 was the only team that exclusively used time series data for the size-100 network challenge, since Team 190 did not submit any results. No results are provided for the GPODE method on size-100 networks either since the algorithm requires a full combinatorial search when no prior knowledge is available, which is computationally intractable for large networks. The OKVAR method is outranked by G1DBN for size-100 E2 in terms of AUPR and for size-100 Y2 with quite comparable AUROC values. It is noticeable that the AUPR values in all rows are rather small (lower than $10 \%$ ) compared to their size-10 counterparts. Such difficult tasks require longer time-series (more time points) and much more available time-series to achieve 
Table 8 Consensus AUROC and AUPR (given in \%) for OKVAR-Prox and OKVAR-Boost run on DREAM3 size-10 and size-100 networks using the DREAM3 original data sets (4 and 46 time series of 21 points for size-10 and size-100 networks respectively)

\begin{tabular}{|c|c|c|c|c|c|c|c|c|c|c|}
\hline & \multicolumn{5}{|c|}{ AUROC } & \multicolumn{5}{|c|}{ AUPR } \\
\hline & E1 & E2 & Y1 & Y2 & Y3 & E1 & E2 & Y1 & Y2 & Y3 \\
\hline \multicolumn{11}{|l|}{ Size-10 } \\
\hline OKVAR-Prox & 81.5 & 78.7 & 76.5 & 70.3 & 75.1 & 32.1 & 50.1 & 35.4 & 37.4 & 39.7 \\
\hline OKVAR-Boost & 85.3 & 74.9 & 68.9 & 65.3 & 69.5 & 58.3 & 53.6 & 28.3 & 26.8 & 44.3 \\
\hline \multicolumn{11}{|l|}{ Size-100 } \\
\hline OKVAR-Prox & 65.4 & 64.0 & 54.9 & 56.8 & 53.5 & 4.6 & 2.6 & 2.3 & 5.0 & 6.3 \\
\hline OKVAR-Boost & 71.8 & 77.2 & 72.9 & 65.0 & 64.3 & 3.6 & 10.7 & 4.2 & 7.3 & 6.9 \\
\hline
\end{tabular}

The numbers in boldface are the maximum values of each column

better results in terms of AUROC and AUPR. Therefore, for size-100 datasets, we applied a pure $\ell_{1}$-norm constraint on model parameters, allowing any $C$ coefficients to be set to 0 rather than a mixed $\ell_{1} / \ell_{2}$-norm regularization that would have been too stringent in terms of data parsimony.

Finally, it is worth noting that OKVAR-Prox would have ranked in the top five and ten, respectively for size-10 and size-100 challenges, while the best results employed knockout/knock-down data in addition to time-series data, the latter being rich in information content (Michailidis 2012).

\subsubsection{Comparison with OKVAR-Boost}

In previous work (Lim et al. 2013), a boosting algorithm (OKVAR-Boost) combining features from $L_{2}$-boosting and randomization-based algorithms was designed. At each boosting iteration, a Hadamard kernel-based OKVAR model was learnt on a random subspace. One main difference between OKVAR-Boost and OKVAR-Prox concerns the learning strategy. While $B$ and $C$ are learnt jointly for the latter, the learning of $B$ and $C$ is decoupled in OKVAR-Boost, meaning that $B$ is firstly estimated by means of a statistical independence test and then $C$ is learnt using an elastic-net regularized loss. A comparison of the two related algorithms is given in Table 8 .

OKVAR-Prox achieves better AUROC values than OKVAR-Boost for size-10 networks, except for the E1 network, while there is no clear winner in terms of AUPR. On size100 inference tasks, OKVAR-Boost which benefits from projections on random subspaces patently outperforms OKVAR-Prox which operates directly in the 100-dimensional space with a limited amount of time points.

\subsection{Climate dataset}

Our second example examines climate data, originally presented in Liu et al. (2010). It contains measurements on climate forcing factors and feedback mechanisms obtained from different databases. We extracted monthly measurements for 12 variables for the year 2002 (i.e. 12 time-points) that include temperature (TMP), precipitation (PRE), vapor (VAP), cloud cover (CLD), wet days (WET), frost days (FRS), Methane (CH4), Carbon Dioxide (CO2), Hydrogen (H2), carbon monoxide (CO), solar radiation (SOL) and aerosols (AER). 
Table 9 Average \pm standard deviation BIC for the climate data set on one location (Northern Texas)

\begin{tabular}{|c|c|c|c|c|}
\hline & & $\lambda_{B}$ & & \\
\hline & & $10^{-2}$ & $10^{-1}$ & 1 \\
\hline$\lambda_{C}$ & $10^{-2}$ & $279.89 \pm 8.20$ & $224.09 \pm 5.76$ & $129.76 \pm 4.23$ \\
\hline & $10^{-1}$ & $311.24 \pm 305.32$ & $115.52 \pm 8.66$ & $60.66 \pm 0.38$ \\
\hline & 1 & $5.63 \times 10^{5} \pm 1.78 \times 10^{6}$ & $5.89 \times 10^{6} \pm 1.86 \times 10^{7}$ & $\mathbf{5 1 . 7 0} \pm \mathbf{0 . 7 9}$ \\
\hline
\end{tabular}

The OKVAR algorithm was run 10 times for each couple of $\left(\lambda_{C}, \lambda_{B}\right)$ hyperparameters. $\lambda_{h}=1$

The measurements were obtained from 125 equally spaced meteorological stations located throughout the United States.

We used an $\ell_{1}$-norm regularized OKVAR-Prox model to identify and explore dependencies between natural and anthropogenic (linked to human activity) factors. We learn a separate causal model for each of the multivariate time series for a specific area in the United States. Therefore, for the sake of exposition clarity, we first consider only a single location in northern Texas.

Similarly to our previous experimental work in Sect. 5.2, we used a grid search in order to set up the hyperparameters of the model. We looked for a combination of $\lambda_{C}$ and $\lambda_{B}$ values that minimize the mean of the Bayesian Information Criterion (BIC) computed over ten independent runs corresponding to ten random initializations of the matrix $B_{0}$, using the data of the northern Texas location. As can be seen in Table 9, we selected the value 1 for both $\lambda_{C}$ and $\lambda_{B}$. With this set up, we subsequently applied Algorithm 2 to the data sets for the remaining 124 locations. A consensus graph was constructed for each location by retaining only a predefined number of edges amongst the best ranked over multiple runs. Given the extreme complexity of the system under study, dependencies may be found between most variables. Therefore, we considered a stringent selection of edges to ease the interpretation of the extracted graph. The level of parsimony (15 edges out of 132) was fixed with the experts in climate data.

For our first experiment focusing on the north Texas dataset, this resulted in a parsimonious directed graph depicted in Fig. 5a.

Most of the edges that OKVAR-Prox identifies are reasonable and supported by external knowledge about the interactions of the underlying variables: specifically, VAP influences CLD since the likelihood of clouds (CLD) appearing increases with vapor concentration (VAP). Vapor (VAP) is also the main natural greenhouse gas on earth, thus corroborating its impact on temperature (TMP). Aerosols (AER) interact with Hydrogen (H2) through atmospheric chemistry and lower the presence of vapor (VAP) by favoring water condensation. Of course, some likely causal links are missing in our final model and one would expect an impact of the concentration of Carbon Dioxide $(\mathrm{CO} 2)$ or Methane $(\mathrm{CH} 4)$ on temperature (TMP). However, note that most of these edges appear in the initial consensus graph and would have been recovered if we set a lower selection threshold.

Since the physical and chemical processes at work in the atmosphere do not change drastically in neighboring locations, the causal graphs learned across the US should exhibit a certain degree of similarity. On the other hand, causal graphs corresponding to distant locations are likely to show topological differences due to regional specificities regarding both climate and human activity. We define the structured similarity $s$ between two graphs $G_{1}$ and $G_{2}$ based on the Hamming Distance between the corresponding adjacency matrices $A_{1}$ and $A_{2}: s\left(G_{1}, G_{2}\right)=1-\frac{1}{d^{2}} \sum_{i, j}\left|A_{1 i j}-A_{2 i j}\right|$. A spectral clustering algorithm using 


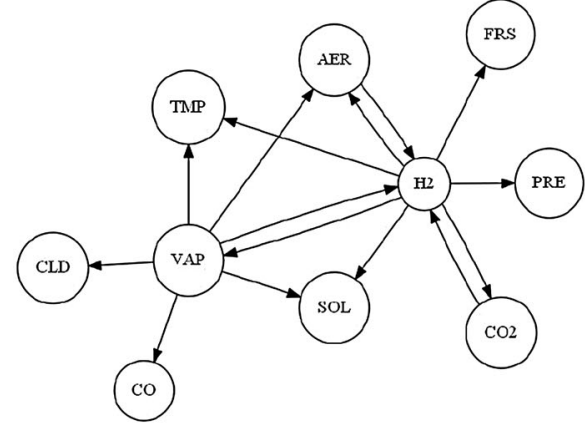

(a)

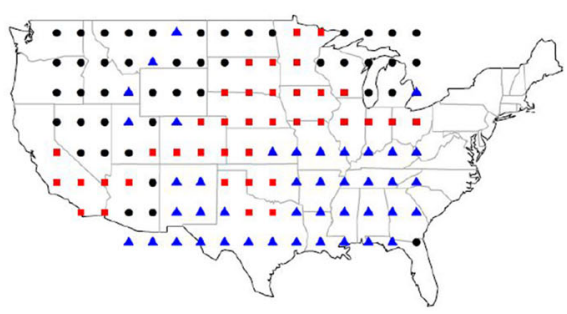

(b)

Fig. 5 a Consensus graph extracted from the climate causal model applied to the north Texas data set, based on 10 independent runs of the OKVAR algorithm. Variables WET and CH4 do not appear since no edges connected them to the rest of the graph. b Partitioning of the causal models learned by OKVAR-Prox, over the United States. All extracted causal graphs have been clustered and labeled using spectral clustering

this similarity matrix with the number of classes set to three was applied; the number of clusters selected a priori was based on the number of hidden variables considered in Liu et al. (2010) for their latent variable model focusing on spatial interactions. Figure 5b shows the labels of the resulting graphs and their corresponding location on a map of the United States. A very clear segmentation of geographical locations emerges, exhibiting the same network structure: the first area (black) includes western and mid-northern locations that have a humid continental climate, another zone (blue) covers developed regions in the South and East, where high levels of $\mathrm{CO} 2$ concentration play a role, while red locations that mostly stretch in the center of the US correspond to less populated areas where human activity factors are less dominant in our model.

\section{Conclusion}

Network inference from multivariate time series represents a key problem in numerous scientific domains. In this paper, we addressed it by introducing and learning a new family of nonlinear vector autoregressive models based on operator-valued kernels. The new models generalize linear vector autoregressive models and benefit from the framework of regularization. To obtain a sparse network estimate, we define appropriate non-smooth penalties on the model parameters and a proximal gradient algorithm to deal with them. Some of the proposed operator-valued kernels are characterized by a positive semi-definite matrix that also plays a role in the network estimation. In this case, an alternating optimization scheme based on two proximal gradient procedures is proposed to learn both kinds of parameters. Results obtained from benchmark size-10 and size-100 biological networks as well as from real climate datasets show very good performance of the OKVAR model. Future extensions include applications of OKVAR to other scientific fields, the use of the model in ensemble methods, and the application of proximal gradient algorithms to other structured output prediction tasks.

Acknowledgments FA-B's work was supported in part by ANR (call SYSCOM-2009, ODESSA project). GM's work was supported in part by NSF Grants DMS-1161838 and DMS-1228164 and NIH Grant 1-R21GM-101719-01-A1. 


\section{References}

Aijo, T., \& Lahdesmaki, H. (2009). Learning gene regulatory networks from gene expression measurements using non-parametric molecular kinetics. Bioinformatics, 25(22), 2937-2944.

Alvarez, M. A., Rosasco, L., \& Lawrence, D. N. (2011). Kernels for vector-valued functions: A review. Technical report, MIT_CSAIL-TR-2011-033.

Auliac, C., Frouin, V., \& Gidrol, X. (2008). Evolutionary approaches for the reverse-engineering of gene regulatory networks: A study on a biologically realistic dataset. BMC Bioinformatics, 9(1), 91.

Baldassarre, L., Rosasco, L., Barla, A., \& Verri, A. (2010). Vector field learning via spectral filtering. In J. Balczar, F. Bonchi, A. Gionis, \& M. Sebag (Eds.), Machine learning and knowledge discovery in databases. Lecture notes in computer science (Vol. 6321, pp. 56-71). Berlin/Heidelberg: Springer.

Beck, A., \& Teboulle, M. (2010). Gradient-based algorithms with applications to signal recovery problems. In D. Palomar \& Y. Eldar (Eds.), Convex optimization in signal processing and communications (pp. 42-88). Cambridge: Cambridge press.

Bolstad, A., Van Veen, B., \& Nowak, R. (2011). Causal network inference via group sparsity regularization. IEEE Trans Signal Process, 59(6), 2628-2641.

Brouard, C., d'Alché Buc, F., \& Szafranski, M. (2011). Semi-supervised penalized output kernel regression for link prediction. In $I C M L-2011$ (pp. 593-600).

Bühlmann, P., \& van de Geer, S. (2011). Statistics for high-dimensional data: Methods, theory and applications. Berlin: Springer.

Caponnetto, A., Micchelli, C. A., Pontil, M., \& Ying, Y. (2008). Universal multitask kernels. The Journal of Machine Learning Research, 9, 1615-1646.

Chatterjee, S., Steinhaeuser, K., Banerjee, A., Chatterjee, S., \& Ganguly, A. R. (2012). Sparse group lasso: Consistency and climate applications. In SDM (pp. 47-58). SIAM/Omnipress

Chou, I., \& Voit, E. O. (2009). Recent developments in parameter estimation and structure identification of biochemical and genomic systems. Mathematical Biosciences, 219(2), 57-83.

Combettes, P. L., \& Pesquet, J. C. (2011). Proximal splitting methods in signal processing. In Fixed-point algorithms for inverse problems in science and engineering. Springer Optimization and Its Applications, Vol. 49, pp. 185-212.

Dinuzzo, F., \& Fukumizu, K. (2011). Learning low-rank output kernels. In Proceedings of the 3rd Asian conference on machine learning, JMLR: Workshop and conference proceedings, Vol. 20.

Dondelinger, F., Lèbre, S., \& Husmeier, D. (2013). Non-homogeneous dynamic bayesian networks with bayesian regularization for inferring gene regulatory networks with gradually time-varying structure. Machine Learning Journal, 90(2), 191-230.

Friedman, N. (2004). Inferring cellular networks using probabilistic graphical models. Science, 303(5659), 799-805.

Gilchrist, S., Yankov, V., \& Zakrajšek, E. (2009). Credit market shocks and economic fluctuations: Evidence from corporate bond and stock markets. Journal of Monetary Economics, 56(4), 471-493.

Hartemink, A. (2005). Reverse engineering gene regulatory networks. Nat Biotechnol, 23(5), 554-555.

Iba, H. (2008). Inference of differential equation models by genetic programming. Information Sciences, $178(23), 4453-4468$.

Kadri, H., Rabaoui, A., Preux, P., Duflos, E., \& Rakotomamonjy, A. (2011). Functional regularized least squares classication with operator-valued kernels. In ICML-2011 (pp 993-1000).

Kolaczyk, E. D. (2009). Statistical analysis of network data: Methods and models: Series in Statistics. Berlin: Springer.

Kramer, M. A., Eden, U. T., Cash, S. S., \& Kolaczyk, E. D. (2009). Network inference with confidence from multivariate time series. Physical Review E, 79(6), 061,916+.

Lawrence, N., Girolami, M., Rattray, M., \& Sanguinetti, G. (Eds.) (2010). Learning and inference in computational systems biology. Cambridge: MIT Press.

Lèbre, S. (2009). Inferring dynamic genetic networks with low order independencies. Statistical Applications in Genetics and Molecular Biology, 8(1), 1-38.

Lim, N., Senbabaoglu, Y., \& Michailidis, G. (2013). OKVAR-Boost: A novel boosting algorithm to infer nonlinear dynamics and interactions in gene regulatory networks. Bioinformatics, 29(11), 1416-1423.

Liu, Y., Niculescu-Mizil, A., \& Lozano, A. (2010). Learning temporal causal graphs for relational time-series analysis. In J. Fürnkranz, \& T. Joachims (Eds.), ICML-2010.

Maathuis, M., Colombo, D., Kalish, M., \& Bühlmann, P. (2010). Predicting causal effects in large-scale systems from observational data. Nature Methods, 7, 247-248.

Margolin, I., \& Nemenman, Aand. (2006). Aracne: An algorithm for the reconstruction of gene regulatory networks in a mammalian cellular context. BMC Bioinformatics, 7(Suppl 1), S7. 
Mazur, J., Ritter, D., Reinelt, G., \& Kaderali, L. (2009). Reconstructing nonlinear dynamic models of gene regulation using stochastic sampling. BMC Bioinformatics, 10(1), 448.

Meinshausen, N., \& Bühlmann, P. (2006). High dimensional graphs and variable selection with the lasso. Annals of Statistics, 34, 1436-1462.

Micchelli, C. A., \& Pontil, M. A. (2005). On learning vector-valued functions. Neural Computation, 17, 177-204.

Michailidis, G. (2012). Statistical challenges in biological networks. Journal of Computational and Graphical Statistics, 21(4), 840-855.

Michailidis, G., \& d'Alché Buc, F. (2013). Autoregressive models for gene regulatory network inference: Sparsity, stability and causality issues. Mathematical Biosciences, 246(2), 326-334.

Morton, R., \& Williams, K. C. (2010). Experimental political science and the study of causality. Cambridge: Cambridge University Press.

Murphy, K. P. (1998). Dynamic bayesian networks: Representation, inference and learning. PhD thesis, Computer Science, University of Berkeley, CA, USA.

Parry, M., Canziani, O., Palutikof, J., van der Linden, P., Hanson, C., et al. (2007). Climate change 2007: Impacts, adaptation and vulnerability. Intergovernmental Panel on Climate Change.

Perrin, B. E., Ralaivola, L., \& Mazurie, A., Bottani, S., Mallet, J., d'Alché-Buc, F. (2003). Gene networks inference using dynamic bayesian networks. Bioinformatics, 19(S2), 38-48.

Prill, R., Marbach, D., Saez-Rodriguez, J., Sorger, P., Alexopoulos, L., Xue, X., et al. (2010). Towards a rigorous assessment of systems biology models: The DREAM3 challenges. PLoS ONE, 5(2), e9202.

Raguet, H., Fadili, \& J., Peyré, G. (2011). Generalized forward-backward splitting. arXiv preprint arXiv:1108.4404.

Richard, E., Savalle, P. A., \& Vayatis, N. (2012). Estimation of simultaneously sparse and low rank matrices. In J. Langford \& J. Pineau (Eds.), ICML-2012 (pp. 1351-1358). New York, NY, USA: Omnipress.

Schaffter, T., Marbach, D., \& Floreano, D. (2011). Genenetweaver: In silico benchmark generation and performance profiling of network inference methods. Bioinformatics, 27(16), 2263-2270.

Senkene, E., \& Tempel'man, A. (1973). Hilbert spaces of operator-valued functions. Lithuanian Mathematical Journal, 13(4), 665-670.

Shojaie, A., \& Michailidis, G. (2010). Discovering graphical granger causality using a truncating lasso penalty. Bioinformatics, 26(18), i517-i523.

Yuan, M., \& Lin, Y. (2006). Model selection and estimation in regression with grouped variables. Journal of the Royal Statistical Society: Series B, 68(1), 49-67.

Zou, C., \& Feng, J. (2009). Granger causality vs. dynamic bayesian network inference: A comparative study. BMC Bioinformatics, 10(1), 122. 